\title{
Rate of convergence: the packing and centered Hausdorff measures of totally disconnected self-similar sets
}

\author{
MARTA LLORENTE, $\mathrm{M}^{a}$ EUGENIA MERA, and MANUEL MORÁN
}

October 18, 2016

\begin{abstract}
In this paper we obtain the rates of convergence of the algorithms given in [13] and [14] for an authomatic computation of the centered Hausdorff and packing measures of a totally disconnected self-similar set. We evaluate these rates empirically through the numerical analysis of three standard classes of self-similar sets, namely, the families of Cantor type sets in the real line and the plane and the class of Sierpinski gaskets. For these three classes and for small contraction ratios, sharp bounds for the exact values of the corresponding measures are obtained and it is shown how these bounds automatically yield estimates of the corresponding measures, accurate in some cases to as many as 14 decimal places. In particular, the algorithms accurately recover the exact values of the measures in all cases in which these values are known by geometrical arguments. Positive results, which confirm some conjetural values given in [13] and [14] for the measures, are also obtained for an intermediate range of larger contraction ratios. We give an argument showing that, for this range of contractin ratios, the problem is inherently computational in the sense that any theoretical proof, such as those mentioned above, might be impossible, so that in these cases, our method is the only available approach. For contraction ratios close to those of the connected case our computational method becomes intractably time consuming, so the computation of the exact values of the packing and centered Hausdorff measures in the general case, with the open set condition, remains a challenging problem.
\end{abstract}

\section{Introduction}

The present paper is part of a program aimed at finding a method for the automatic computation of metric measures, such as the packing or Hausdorff measure, of a given fractal set. In particular, we obtain the rates of convergence of the algorithms given in [13] and [14] for computing the centered Hausdorff and packing measures, respectively, of a totally disconnected self-similar set. It is important to note that, although the convergence of these algorithms was shown in [13] and [14] without establishing their rates of convergence, the outputs of the algorithms were still useful for obtaining conjectural values of the measures. Using results presented in this note we can prove these conjectures (see Sections 2.3 and 3.3).

Recall that a totally disconnected self-similar set associated to a system $\Psi=\left\{f_{1}, f_{2, \ldots}, f_{m}\right\}$ of contracting similitudes of $\mathbb{R}^{n}$ is a compact non-empty set $E \subset \mathbb{R}^{n}$ such that $E=\bigcup_{i=1}^{m} f_{i}(E)$ and satisfying

$$
f_{i}(E) \cap f_{j}(E)=\emptyset \forall i \neq j, \quad i, j \in\{1, \ldots, m\}=: M .
$$

The last condition implies the Open Set Condition (OSC, see [9]), and it is known as the Strong Separation Condition $(S S C)$. Throughout the paper we assume the system $\Psi$ satisfies the $S S C$ and write

$$
c:=\min _{\substack{i, j \in M \\ i \neq j}} d_{\inf }\left(f_{i}(E), f_{j}(E)\right)>0,
$$

where $d_{\mathrm{inf}}\left(f_{i}(E), f_{j}(E)\right)$ is the distance between $f_{i}(E)$ and $f_{j}(E)$. The similarity ratio of $f_{i} \in \Psi$ is denoted by $r_{i} \in(0,1)$, and we write

$$
r_{\min }:=\min _{i=1, \ldots, m} r_{i} \quad \text { and } \quad r_{\max }:=\max _{i=1, \ldots, m} r_{i} .
$$

Both algorithms are based on the self-similar tiling principle stated in [17] for self-similar sets satisfying the OSC. In [17] it was shown that if $B$ is any closed subset (or tile) of a self-similar set $E$ such that $\mu(B)>0$, where $\mu$ is the invariant measure (see (8)), then $E$ can be tiled, without any loss of $\mu$-measure, by a countable collection of tiles that are images of $B$ under similitudes. Recall from [9] that, for self-similar sets satisfying the OSC, the measure $\mu$ is a multiple of any scaling measure, and in particular of the packing, Hausdorff, or centered Hausdorff measure.

Taking an appropriate initial tile $B$ (one with minimal spherical density in the case of the packing measure, and with maximal spherical density in the case of the Hausdorff measure; see Remark 4) we obtain both an optimal packing or covering [11], and the exact value of the corresponding measure. Our method requires a particular form of the separation 
condition, the SSC, in order to make the computation of the metric measures feasible (see Section 4 for a discussion of the computability of metric measures satisfying the OSC).

Metric measures suitable for studying the size of sets of Lebesgue measure zero in $\mathbb{R}^{n}$, such as the Hausdorff, packing, and centered Hausdorff measure $\left(H^{s}, P^{s}\right.$, and $C^{s}$, respectively) have been studied intensively in recent years. However, the challenging problem of finding systematic methods for computing the values of these measures for a general fractal set remains open. Much effort has been made in this direction, and exact values and bounds for the measures of some fractal sets are known already (see [1]-[10], [11]-[14], [16], [17], [21], [25], and the references therein).

In this direction, the algorithms presented in [13] and [14] can be seen as the first steps towards the systematic computation of the centered Hausdorff and packing measures of a self-similar set. These algorithms yield estimates of the corresponding measures for a wide class of self-similar sets, taking as input the list of contracting similitudes associated with the given set. It is important to note that in some cases, such as for the class of Sierpinski gaskets with dimension less than or equal to one, the packing measure algorithm has been useful not only for estimating the value of $P^{s}$ on each particular set in the class, but also for finding a formula for the packing measure of an arbitrary member of the class. As shown in [14, Theorem 2], the information provided by the algorithm can then be used to prove the formula. However, in many other cases, such as for some plane self-similar sets of dimension greater than one, the absence of the corresponding formula means that it is desirable to know the accuracy of the numerical results obtained from the algorithms. In [13] the centered Hausdorff measure algorithm was implemented for some sets whose centered Hausdorff measures were available in the literature and some other sets whose centered Hausdorff measures were still unknown. It is remarkable that, in the first case, the optimal values were attained at early iterations and, in the second case, the algorithm yielded conjectural values that could be proved with the methods developed in [14]. However, the rate of convergence of neither algorithm was known. This is the problem we solve in this paper and the content of the next two main theorems.

Theorem 1 Suppose that the system $\Psi=\left\{f_{1}, \ldots, f_{m}\right\}$ satisfies the SSC. Then, for every $k \in \mathbb{N}^{+}$such that $c-3 R r_{\max }^{k}-$ $2 R r_{\max }^{k+1}>0$ and every $\tilde{M}_{k}$ as in (18) there holds

$$
\left|P^{s}(E)-\tilde{M}_{k}\right| \leq \varepsilon_{k}
$$

where

$$
\varepsilon_{k}:=\frac{s 2^{s+1} R Q}{r_{\min }^{s q_{k}}} r_{\max }^{k}
$$

$s=\operatorname{dim}_{H}(E), q_{k} \in \mathbb{N}^{+}$is such that $R r_{\max }^{q_{k}} \leq c-R 2 r_{\max }^{k+1}-2 R r_{\max }^{k}<R r_{\max }^{q_{k}-1}$, and

$$
Q:=\left\{\begin{array}{c}
\left(\frac{c}{r_{\min }}\right)^{s-1} \text { if } s \geq 1, \\
\left(c-2 R r_{\max }^{k}-2 R r_{\max }^{k+1}\right)^{s-1} \text { if } s<1 .
\end{array}\right.
$$

Theorem 2 Suppose that the system $\Psi=\left\{f_{1}, \ldots, f_{m}\right\}$ satisfies the SSC. Then, for every $k \in \mathbb{N}^{+}$and every $\tilde{m}_{k}$ given by (39), there holds

$$
\left|C^{s}(E)-\tilde{m}_{k}\right| \leq \epsilon_{k}
$$

where

$$
\epsilon_{k}:=\frac{s 2^{s+1} R \mathcal{Q}}{r_{\min }^{q s}} r_{\max }^{k}
$$

$s=\operatorname{dim}_{H}(E), q \in \mathbb{N}^{+}$is such that $R r_{\text {max }}^{q} \leq c<R r_{\text {max }}^{q-1}$, and

$$
\mathcal{Q}:=\left\{\begin{array}{l}
R^{s-1} \text { if } s \geq 1, \\
c^{s-1} \text { if } s<1 .
\end{array}\right.
$$

Here, $\operatorname{dim}_{H}(E)$ and $R$ stand for the Hausdorff dimension and the diameter of the self-similar set $E$.

As discussed in Sections 2.3 and 3.3, one of the most important features of Theorems 1 and 2 is that they provide sharp bounds for the exact values of the corresponding measures. Moreover, these bounds yield automatically estimates of the corresponding measures, accurate in some cases to as many as 14 decimal places. In the difficult case of self-similar sets having dimensions greater than one, for which less is known, we give examples with five decimal place accuracy. For instance, applying Theorem 1 to the family of Sierpinski gaskets $\left\{S_{r}\right\}$ with $\operatorname{dim}_{H}\left(S_{r}\right)=-\frac{\log 3}{\log r}$ (see (26) for a definition), yields $P^{s}\left(S_{0.37}\right) \simeq 3.8728$ (see Table 3 ) and $P^{s}\left(S_{0.42}\right) \simeq 3.62$. We also get $P^{s}\left(K_{\frac{4}{10}}\right) \simeq 5.27$, where $K_{\frac{4}{10}}$ is the plane Cantor set of dimension $-\frac{\log 4}{\log 0.4}$. To our knowledge none of these estimates were previously known.

However, the most important consequence of the combination of Theorems 1 and 2 with the algorithms given in [13] and [14] is that it automatically provides an approximation to the value of the measure of any self-similar set satisfying the SSC. We remark that the precision of the results depends on the size of the contraction ratios. Namely, the accuracy 
achieved improves as the contraction ratios decrease (see [13], [14] and Section 4 below for a detailed discussion). In particular, the examples given in Sections 2.3 and 3.3 show that the algorithms accurately recover the known values of these measures for sets with dimension less than one. Moreover, the results presented in this article serve to rule out certain potential formulas for some classes of self-similar sets.

The paper is divided into two main sections, one devoted to the packing measure and the other to the centered Hausdorff measure. In each case, we first recall the relevant algorithm from [13] or [14], although in the case of the centered Hausdorff measure we give some improvements to the algorithm. This is done in Sections 2.1 and 3.1. Then, we prove Theorems 1 and 2 at the ends of Sections 2.2 and 3.2, respectively. It is remarkable that these proofs do not use the convergence of the corresponding algorithms, so the present note provides shorter alternative proofs of their convergence. Finally, Sections 2.3 and 3.3 are devoted to analyzing the results obtained by applying Theorems 1 and 2 to the examples given in [13] and [14]. These numerical experiments have a twofold purpose. On the one hand they illustrate the theoretical results, showing how the algorithms perform in practice. On the other hand they offer quite complete information, previously unavailable in the literature, on the exact values of the packing and centered Hausdorff measures of the self-similar sets in three of the most classic families of self-similar sets, namely, the central Cantor sets in the line, the Sierpinski gaskets, and the Cantor sets in the unit square. Finally, in Section 4 we discuss the computability of metric measures on self-similar sets in view of the results obtained in this paper.

Notational Convention 3 We denote the open and closed ball with center $x$ and radius $r$ by $B(x, d)=\left\{y \in \mathbb{R}^{n}\right.$ : $|x-y|<d\}$ and $\bar{B}(x, d)=\left\{y \in \mathbb{R}^{n}:|x-y| \leq d\right\}$, respectively. We use the notation $\partial B(x, d)$ for the boundary of $B(x, d)$. Given $A \subset \mathbb{R}^{n}$, we write $|A|$ for the diameter of $A$ and $A^{c}=\left\{x \in \mathbb{R}^{n}: x \notin A\right\}$ for the complement of $A$.

We write $s$ for the similarity dimension of $E$, i.e., the unique solution $s$ of $\sum_{i=1}^{m} r_{i}^{s}=1$. Sometimes we will refer to $s$ as the Hausdorff dimension, $\operatorname{dim}_{H}(E)$, of $E$, since the similarity and Hausdorff dimension coincide when $E$ is a totally disconnected self-similar set, as in the present note.

For the code space we use the following notation. Let $M:=\{1, \ldots, m\}$ and

$$
M^{k}=\left\{\mathbf{i}_{k}=\left(i_{1}, \ldots, i_{k}\right): i_{j} \in M \quad \forall j=1, \ldots, k\right\} .
$$

Given $\mathbf{i}_{k}=i_{1} i_{2} \ldots i_{k} \in M^{k}$, we write $f_{\mathbf{i}_{k}}$ for the similitude $f_{\mathbf{i}_{k}}=f_{i_{1}} \circ f_{i_{2}} \circ \cdots \circ f_{i_{k}}$ with similarity ratio $r_{\mathbf{i}_{k}}=r_{i_{1}} r_{i_{2}} \ldots r_{i_{k}}$, and, given $A \subset \mathbb{R}^{n}$, we write $A_{\mathbf{i}_{k}}=f_{\mathbf{i}_{k}}(A)$, and refer to the sets $E_{\mathbf{i}_{k}}=f_{\mathbf{i}_{k}}(E)$ as the cylinder sets of generation $k$. In particular, the sets $E_{i}=f_{i}(E), i \in M$, are called basic cylinder sets.

We denote by $\mu$ the natural probability measure, or normalized Hausdorff measure, defined on the ring of cylinder sets by

$$
\mu\left(E_{\mathbf{i}}\right)=r_{\mathbf{i}}^{s}, \quad \forall \mathbf{i} \in \cup_{k=1}^{\infty} M^{k},
$$

and then extended to Borel subsets of $E$ (see [9]).

Remark 4 With the above notation, the idea underlying the estimation of $P^{s}(E)$ and $C^{s}(E)$ can be summarized as follows: Find the minimum and the maximum of the spherical densities $\frac{\mu(B(x, r))}{(2 r)^{s}}$ on suitable families of balls. The inverse of the minimum is the desired estimate for $P^{s}(E)$ and the inverse of the maximum is that for $C^{s}(E)$. Furthermore, these estimates give valuable additional information about the behavior of $\mu$. For, if we let

$$
\text { Spec }=\left\{\lim _{k \rightarrow \infty} \frac{\mu\left(B\left(x, r_{k}\right)\right)}{\left(2 r_{k}\right)^{s}}: x \in E \text { and } \lim _{k \rightarrow \infty} r_{k}=0\right\}
$$

be the full range of limiting values of the spherical densities of $\mu$ on balls, then the interval $\left[P^{s}(E)^{-1}, C^{s}(E)^{-1}\right]$ is the minimal interval that contains Spec or spectral range of the density of $\mu$ (see [12] and [17]).

\section{Packing measure}

The packing measure of a compact set $A$ with finite packing premeasure can be defined by

$$
P^{s}(A)=\lim _{\delta \rightarrow 0} P_{\delta}^{s}(A),
$$

where

$$
P_{\delta}^{s}(A)=\sup \left\{\sum_{i=1}^{\infty}\left|B_{i}\right|^{s}:\left|B_{i}\right| \leq \delta, i=1,2,3, \ldots\right\}
$$

is a set function nondecreasing with respect to $\delta$ and the supremum is taken over all countable collections of disjoint Euclidean balls centered in $A$ and having diameters smaller than $\delta$ (see [7]). Recall that, as explained in [19]-[23], a two-stage definition is needed for general Euclidean sets.

In the specific case of self-similar sets much effort has been made to find a simplified definition of $P^{s}$ suitable for computation. In [17] it was shown how the above one-stage definition allows a characterization of $P^{s}$ in terms of density functions which later facilitated tackling the computability problem algorithmically (see [11] and [24]). Next, we see that this characterization is also central for proving the rate of convergence of the packing measure algorithm given by Theorem 1. 


\subsection{Previous results: Packing measure algorithm}

We begin with the following formula for the packing measure used in [14] as a starting point for the construction of an efficient algorithm. For a self-similar set $E$ satisfying the SSC,

$$
P^{s}(E)=\max \left\{h(x, d):(x, d) \in E \times\left[c, \frac{c}{r_{\min }}\right]\right\},
$$

where $h(x, d):=\frac{(2 d)^{s}}{\mu(B(x, d))}$. In $\left[14\right.$, Theorem 1], there was proved the more general characterization of $P^{s}(E)$ as

$$
\begin{aligned}
P^{s}(E) & =\max \{h(x, d): x \in E, d \leq a\} \\
& =\max \left\{h(x, d): x \in E, a r_{\min } \leq d \leq a\right\},
\end{aligned}
$$

where $a \in\left(0, \frac{c}{r_{\min }}\right]$ (see (1), (2), and (3), for the meaning of the notation used here). The reason for choosing $a=\frac{c}{r_{\min }}$ in

(9) is to increase the efficiency of the algorithm by both reducing the cardinality of the set of balls on which the maximum is to be computed and increasing the radii of these balls. However, for convenience, in the proof of Theorem 1 we shall use (11) in the form

$$
P^{s}(E)=\max \left\{h(x, d): x \in E, b \leq d \leq \frac{b}{r_{\min }}\right\}
$$

where $b \in(0, c]$.

Next, we recall the algorithm developed in [14] for computing the value of $P^{s}(E)$ via approximations of the maximal value of $h(x, d)$.

Algorithm 5 (Packing measure algorithm) Input of the Algorithm: The system, $\Psi$, of contracting similitudes and $k_{\max }$, the iteration on which the algorithm's run was stopped.

Let $k \leq k_{\max }$ such that $c-2 R r_{\max }^{k}-2 R r_{\max }^{k+1}>0$.

1. Construction of $\mathbf{A}_{k}$. Let $A_{1}=\cup_{i \in M}\left\{x \in \mathbb{R}^{n}: f_{i}(x)=x\right\}$ be the set consisting of the $m$ fixed points of the similitudes in $\Psi$. For every $k \in \mathbb{N}^{+}$, let $A_{k}=S \Psi\left(A_{k-1}\right)$ be the set formed by the $m^{k}$ points obtained by applying $S \Psi(x)=\bigcup_{i \in M} f_{i}(x)$ to each of the $m^{k-1}$ points of $A_{k-1}$.

Notational Convention 6 For every $x \in A_{k}$ we denote by $\mathbf{i}_{k}^{x}=i_{1}^{x} \ldots i_{k}^{x} \in M^{k}$ the unique sequence of length $k$ such that $x=f_{\mathbf{i}_{k}^{x}}(y)$ for some $y \in A_{1}$. Then $E_{\mathbf{i}_{k}^{x}}=f_{\mathbf{i}_{k}^{x}}(E)$ denotes the unique cylinder set of generation $k$ such that $x \in E_{\mathbf{i}_{k}^{x}}$. Observe that

$$
x \in A_{k} \backslash A_{k-1} \Longleftrightarrow i_{k}^{x} \neq i_{k-1}^{x} \text {. }
$$

\section{Generation of the list of distances.}

This step consists in computing the set

$$
\Delta_{k}:=\left\{\operatorname{dist}(x, y):(x, y) \in A_{k} \times A_{k}\right\}
$$

of distances between pairs of points in $A_{k}$. It is important to note that $\Delta_{k} \subset \Delta_{k+1}$ since, by construction, $A_{k} \subset A_{k+1}$. Hence, the computation of the set $\Delta_{k-1}$ of distances should be avoided in the construction of $\Delta_{k}$. Therefore, we shall calculate only those distances $\operatorname{dist}(x, y) \in \Delta_{k}$ where

$$
i_{k}^{x} \neq i_{k-1}^{x} \text { or } i_{k}^{y} \neq i_{k-1}^{y} .
$$

For every $k \in \mathbb{N}^{+}$we write $\Delta_{k}^{0}:=\left\{\operatorname{dist}(x, y) \in \Delta_{k}: i_{k}^{x} \neq i_{k-1}^{x}\right.$ or $\left.i_{k}^{y} \neq i_{k-1}^{y}\right\}$ with $\Delta_{1}^{0}=\Delta_{1}$ and we write $\Delta_{k}=\Delta_{k}^{0} \cup \Delta_{k-1}$.

Henceforth, we assign the code $\left(\mathbf{i}_{k}^{x}, \mathbf{i}_{k}^{y}\right)$ to each dist $(x, y) \in \Delta_{k}$ and refer to $\left(\mathbf{i}_{k}^{x}, \mathbf{i}_{k}^{y}\right)$ as the $k$-address of $\operatorname{dist}(x, y)$. Observe that the $(k+1)$-address of dist $(x, y) \in \Delta_{k}$ is

$$
\left(\mathbf{i}_{k}^{x} i_{k}^{x}, \mathbf{i}_{k}^{y} i_{k}^{y}\right)=\left(i_{1}^{x} \ldots i_{k}^{x} i_{k}^{x}, i_{1}^{y} \ldots i_{k}^{y} i_{k}^{y}\right) .
$$

3. Construction of $\mu_{k}$. Given $k \in \mathbb{N}^{+}$, set

$$
\mu_{k}(x)=r_{\mathbf{i}_{k}^{x}}^{s} \forall x \in A_{k} .
$$

Then,

$$
\mu_{k}=\sum_{x \in A_{k}} r_{\mathbf{i}_{k}^{x}}^{s} \delta_{x}
$$

is a discrete probability measure supported on the $m^{k}$ points of $A_{k}$. 


\section{Construction of $\tilde{M}_{k}$.}

Given $x \in A_{k}$ :

4.1 Rank in increasing order the distances $d \in \Delta_{k}$ containing the letter $\mathbf{i}_{k}^{x}$ in their addresses and such that $d \leq \frac{c}{r_{\min }}$.

4.2 Let $0=d_{1}^{x} \leq d_{2}^{x} \leq \cdots \leq d_{m_{x}}^{x}$ be the list of ordered distances, where $m_{x} \in\left\{1, \ldots, m^{k}\right\}$. For every $j \in$ $\left\{1, \ldots, m_{x}\right\}$, let $t_{j} \leq j$ be such that $d_{j}^{x}=d_{j-1}^{x}=\cdots=d_{t_{j}}^{x} \neq d_{t_{j}-1}^{x}$. Then

$$
\mu_{k}\left(B\left(x, d_{j}^{x}\right)\right):=\sum_{q=1}^{t_{j}-1} r_{\mathbf{i}_{k}^{x}}^{S},
$$

where $x_{q} \in A_{k}$ is the point chosen for calculating the distance $d_{q}^{x}=\operatorname{dist}\left(x, x_{q}\right)$, for every $q=1, \ldots, j$ and $\mu_{k}\left(B\left(x, d_{1}^{x}\right)\right)=0$.

In the particular case when $r_{i}=r$ for all $i \in M$, (16) simplifies to

$$
\mu_{k}\left(B\left(x, d_{j}^{x}\right)\right)=\frac{t_{j}-1}{m^{k}} .
$$

Compute

$$
h_{k}\left(x, d_{j}^{x}\right):=\frac{\left(2 d_{j}^{x}\right)^{s}}{\mu_{k}\left(B\left(x, d_{j}^{x}\right)\right)}=\frac{\left(2 d_{j}^{x}\right)^{s}}{\sum_{q=1}^{t_{j}-1} r_{\mathbf{i}_{k}^{x_{q}}}^{s}}
$$

only for those distances $d_{j}^{x}$ in the list satisfying

$$
0<c-2 R r_{\max }^{k}-2 R r_{\max }^{k+1} \leq d_{j}^{x}
$$

4.3 Find the maximum

$$
M_{k}(x):=\max \left\{h_{k}\left(x, d_{j}^{x}\right): c-2 R r_{\max }^{k}-2 R r_{\max }^{k+1} \leq d_{j}^{x} \leq \frac{c}{r_{\min }}\right\}
$$

of the values computed in 4.2.

4.4 Repeat steps 4.1-4.3 for each $x \in A_{k}$.

4.5 Take the maximum

$$
\tilde{M}_{k}:=\max \left\{M_{k}(x): x \in A_{k}\right\}
$$

of the $m^{k}$ values computed in step 4.4 .

Observe that, for some $\left(\tilde{x}_{k}, \tilde{y}_{k}\right) \in A_{k} \times A_{k}$,

$$
\begin{aligned}
\tilde{M}_{k} & :=h_{k}\left(\tilde{x}_{k}, \operatorname{dist}\left(\tilde{x}_{k}, \tilde{y}_{k}\right)\right) \\
& =\max \left\{h_{k}(x, \operatorname{dist}(x, y)):(x, y) \in A_{k} \times A_{k} \text { and } c-2 R r_{\max }^{k}-2 R r_{\max }^{k+1} \leq \operatorname{dist}(x, y) \leq \frac{c}{r_{\min }}\right\} .
\end{aligned}
$$

Notational Convention 7 In what follows we use the following notation. We denote by $D_{k}^{x}$ the set of distances selected in steps 4.1 and 4.2, and we let $D_{k}:=\cup_{x \in A_{k}} D_{k}^{x}$. We refer to $D_{k}^{x}$ as the set of admissible distances for $x \in A_{k}$. Note that

$$
D_{k}^{x} \subset D_{k} \subset\left[c-2 R r_{\max }^{k}-2 R r_{\max }^{k+1}, \frac{c}{r_{\min }}\right] .
$$

It is important to note that the balls admissible in the algorithm have radii in the interval $\left[c-2 R r_{\max }^{k}-2 R r_{\max }^{k+1}, \frac{c}{r_{\min }}\right] \supset$ $\left[c, \frac{c}{r_{\min }}\right]$ (see (9)). This containment helps in comparing the densities giving the packing measure with those computed by the algorithm (see Section 2.2).

\subsection{Rate of convergence for the packing measure algorithm}

This section is devoted to proving Theorem 1. One of the difficulties one needs to overcome to show the rate of convergence (4) is to obtain a comparison between the measures $\mu$ and $\mu_{k}$ of a given ball (see (8) and (15)). Note that to obtain a bound for $\left|P^{s}(E)-\tilde{M}_{k}\right|$ we need to compare the densities $h(x, d)$ given in (12) with those given in (19). The following lemmas show that it is possible to construct the approximating balls needed for such a comparison.

Lemma 8 For every $k \in \mathbb{N}^{+}$and $(x, d) \in E \times(0, \infty)$ such that $\partial B(x, d) \cap E \neq \emptyset$, there exists $\left(x^{\prime}, d^{\prime}\right) \in A_{k} \times[d-$ $\left.2 R r_{\max }^{k}, d+2 R r_{\max }^{k}\right]$ such that 
(i) $\left|x-x^{\prime}\right| \leq R r_{\max }^{k}$,

(ii) $d^{\prime}=\left|x^{\prime}-y\right|$ for some $y \in A_{k}$,

(iii) $\mu(B(x, d)) \geq \mu_{k}\left(B\left(x^{\prime}, d^{\prime}\right)\right)$.

Proof. Let $k \in \mathbb{N}^{+}$and $(x, d) \in E \times(0, \infty)$ be such that $\partial B(x, d) \cap E \neq \emptyset$. Take $x^{\prime}$ to be the unique point in $A_{k} \cap E_{\mathbf{i}_{k}^{x}}$. Then (i) holds.

Set $L:=\left\{y \in A_{k}: E_{\mathbf{i}_{k}^{y}} \cap B^{c}(x, d) \neq \emptyset\right\}$ and let $d^{\prime}:=\min \left\{\left|x^{\prime}-y\right|: y \in L\right\}$. Observe that the assumption $\partial B(x, d) \cap E \neq \emptyset$ implies that $L \neq \emptyset$. Moreover, we can assume that $d^{\prime} \neq 0$ since, otherwise, $x^{\prime} \in L, d \leq R r_{\text {max }}^{k}$, and $\mu_{k}\left(B\left(x^{\prime}, d^{\prime}\right)\right)=0($ see step 4.2 in Algorithm 5) and the lemma holds true.

In order to check the inequality $d^{\prime} \leq d+2 R r_{\max }^{k}$, take $z \in \partial B(x, d) \cap E$ and $y \in A_{k} \cap E_{\mathbf{i}_{k}^{z}}$. Then, $y \in L$ and the triangle inequality together with (i) imply

$$
d^{\prime} \leq\left|x^{\prime}-y\right| \leq\left|x-x^{\prime}\right|+|x-z|+|y-z| \leq d+2 R r_{\max }^{k} .
$$

The inequality $d^{\prime} \geq d-2 R r_{\max }^{k}$ follows from (i) and the triangle inequality by taking $y \in L$ such that $d^{\prime}=\left|x^{\prime}-y\right|$ and $z \in E_{\mathbf{i}_{k}^{y}} \cap B^{c}(x, d)$, since then

$$
d \leq|x-z| \leq\left|x-x^{\prime}\right|+\left|x^{\prime}-y\right|+|y-z| \leq d^{\prime}+2 R r_{\max }^{k} .
$$

Finally, (iii) holds because for all $y \in B\left(x^{\prime}, d^{\prime}\right) \cap A_{k}$ we have that $y \notin L$, whence $E_{\mathbf{i}_{k}^{y}} \subset B(x, d)$. This, in turn, implies

$$
\begin{aligned}
\mu(B(x, d)) & =\mu\left(\left\{E_{\mathbf{i}_{k}}: E_{\mathbf{i}_{k}} \subset B(x, d)\right\}\right)+\mu\left(\left\{E_{\mathbf{i}_{k}} \cap B(x, d): E_{\mathbf{i}_{k}} \cap B^{c}(x, d) \neq \emptyset\right\}\right) \\
& \geq \mu_{k}\left(\left\{y \in A_{k}: E_{\mathbf{i}_{k}^{y}} \subset B(x, d)\right\}\right) \geq \mu_{k}\left(B\left(x^{\prime}, d^{\prime}\right)\right),
\end{aligned}
$$

which concludes the proof of (iii).

Lemma 9 For every $k \in \mathbb{N}^{+}$and $(x, d) \in A_{k} \times D_{k}^{x}$, there exists $d^{\prime} \in\left[d-R r_{\max }^{k}, d\right]$ such that $\mu_{k}(B(x, d)) \geq \mu\left(B\left(x, d^{\prime}\right)\right)$.

Proof. Let $k \in \mathbb{N}^{+}$and $(x, d) \in A_{k} \times D_{k}^{x}$ (see Notational Convention 7 ). Set $d^{\prime}:=\min \left\{\operatorname{dist}\left(x, E_{\mathbf{i}_{k}}\right): E_{\mathbf{i}_{k}} \cap B^{c}(x, d) \neq \emptyset\right\}$. Note that, by definition of $D_{k}^{x}, \partial B(x, d) \cap A_{k} \neq \emptyset$, and therefore $d^{\prime} \leq d$. In order to show $d^{\prime} \geq d-R r_{\max }^{k}$, choose $y \in E$ such that $d^{\prime}=|x-y|$ and $z \in B^{c}(x, d) \cap E_{\mathbf{i}_{k}^{y}}$. Then, by the triangle inequality,

$$
d \leq|x-z| \leq|x-y|+|y-z| \leq d^{\prime}+R r_{\max }^{k} .
$$

Finally, the inequality $\mu_{k}(B(x, d)) \geq \mu\left(B\left(x, d^{\prime}\right)\right)$ follows because $B\left(x, d^{\prime}\right) \cap E \subset\left\{y \in E: E_{\mathbf{i}_{k}^{y}} \subset B(x, d)\right\}$ and, therefore

$$
\begin{aligned}
\mu\left(B\left(x, d^{\prime}\right)\right) & \leq \mu\left(\left\{y \in E: E_{\mathbf{i}_{k}^{y}} \subset B(x, d)\right\}\right) \\
& =\mu_{k}\left(\left\{y \in A_{k}: E_{\mathbf{i}_{k}^{y}} \subset B(x, d)\right\}\right) \leq \mu_{k}(B(x, d)) .
\end{aligned}
$$

\section{Remark 10}

1. Observe that, if $q_{k} \in \mathbb{N}^{+}$is as in Theorem 1, then $E_{\mathbf{i}_{q_{k}}^{x}} \subset B(x, d)$ for any $(x, d) \in A_{k} \times D_{k}^{x}$ and, therefore,

$$
\mu_{k}(B(x, d)) \geq \mu\left(E_{\mathbf{i}_{q_{k}}^{x}}\right) \geq r_{\min }^{q_{k} s} .
$$

2. In the proof of Theorem 1 we shall use the following result from [14]: Given $a \in\left(0, \frac{c}{r_{\min }}\right]$ and $\left(x_{0}, d_{0}\right) \in E \times\left[a r_{\min }, a\right]$ such that $P^{s}(E)=h\left(x_{0}, d_{0}\right)=\frac{\left(2 d_{0}\right)^{s}}{\mu\left(B\left(x_{0}, d_{0}\right)\right)}$, then

$$
\partial B\left(x_{0}, d_{0}\right) \cap E \neq \emptyset .
$$

Now we are ready to prove our main result.

Proof of Theorem 1. We divide the proof into two cases: $P^{s}(E) \geq \tilde{M}_{k}$ and $P^{s}(E) \leq \tilde{M}_{k}$.

Let $k \in \mathbb{N}^{+}$. Suppose first that $P^{s}(E) \geq \tilde{M}_{k}$ and let $\mathcal{B}:=\left\{(x, d): x \in E\right.$ and $\left.d \in\left[c-2 R r_{\max }^{k+1}, \frac{c-2 R r_{\max }^{k+1}}{r_{\min }}\right]\right\}$. Take $(\tilde{x}, \tilde{d}) \in \mathcal{B}$ such that

$$
h(\tilde{x}, \tilde{d})=P^{s}(E)=\max \{h(x, d):(x, d) \in \mathcal{B}\}
$$

(see (11)). By (22) we know that $\partial B(\tilde{x}, \tilde{d}) \cap E \neq \emptyset$, so we can apply Lemma 8 with $(x, d)=(\tilde{x}, \tilde{d})$ and take $\left(x^{\prime}, d^{\prime}\right) \in$ $A_{k} \times\left[\tilde{d}-2 R r_{\max }^{k}, \tilde{d}+2 R r_{\max }^{k}\right] \subset A_{k} \times\left[c-2 R r_{\max }^{k}-2 R r_{\max }^{k+1}, \frac{c}{r_{\min }}\right]$. It is then clear, by (20) that $0<d^{\prime} \in D_{k}^{x^{\prime}}$, and hence 
$B\left(x^{\prime}, d^{\prime}\right)$ is an admissible ball for the algorithm. This, together with (19), (21), (23), (iii) of Lemma 8, and the mean value theorem, gives

$$
\begin{aligned}
P^{s}(E)-\tilde{M}_{k} & \leq \frac{(2 \tilde{d})^{s}}{\mu(B(\tilde{x}, \tilde{d}))}-\frac{\left(2 d^{\prime}\right)^{s}}{\mu_{k}\left(B\left(x^{\prime}, d^{\prime}\right)\right)} \\
& \leq 2^{s} \frac{(\tilde{d})^{s}-\left(\tilde{d}-2 R r_{\max }^{k}\right)^{s}}{\mu_{k}\left(B\left(x^{\prime}, d^{\prime}\right)\right)} \leq \frac{s 2^{s+1} R Q}{r_{\min }^{s q_{k}}} r_{\max }^{k}
\end{aligned}
$$

where $Q$ is as in (5).

Now, suppose that $P^{s}(E) \leq \tilde{M}_{k}$ and let $(\tilde{x}, \tilde{d}) \in A_{k} \times D_{k}^{\tilde{x}}$ be such that $\tilde{M}_{k}=h_{k}(\tilde{x}, \tilde{d})$. Take $d^{\prime}$ as in Lemma 9 with $(x, d)=(\tilde{x}, \tilde{d})$. Then $\mu_{k}(B(\tilde{x}, \tilde{d})) \geq \mu\left(B\left(\tilde{x}, d^{\prime}\right)\right)$ with $\left(\tilde{x}, d^{\prime}\right) \in A_{k} \times\left[\tilde{d}-R r_{\max }^{k}, \tilde{d}\right] \subset E \times\left[c-3 R r_{\max }^{k}-2 R r_{\max }^{k+1}, \frac{c}{r_{\min }}\right]$. Therefore, by (12), with $b=c-3 R r_{\max }^{k}-2 R r_{\max }^{k+1}>0$, we obtain

$$
h\left(\tilde{x}, d^{\prime}\right) \leq P^{s}(E)=\max \left\{h(x, d): x \in E, d \in\left[c-3 R r_{\max }^{k}-2 R r_{\max }^{k+1}, \frac{c}{r_{\min }}\right]\right\} .
$$

All this, together with the mean value theorem, gives

$$
\begin{aligned}
& \tilde{M}_{k}-P^{s}(E) \leq \frac{(2 \tilde{d})^{s}}{\mu_{k}(B(\tilde{x}, \tilde{d}))}-\frac{\left(2 d^{\prime}\right)^{s}}{\mu\left(B\left(\tilde{x}, d^{\prime}\right)\right)} \\
& \leq 2^{s} \frac{\tilde{d}^{s}-\left(\tilde{d}-2 R r_{\max }^{k}\right)^{s}}{\mu_{k}(B(\tilde{x}, \tilde{d}))} \leq \frac{s 2^{s+1} R Q}{r_{\min }^{s q_{k}}} r_{\max }^{k}
\end{aligned}
$$

where $Q$ is as in (5). This concludes the proof of the theorem.

\section{$2.3 \quad$ Examples}

Algorithm 5 was tested in [14] with various different classes of self-similar sets, including those previously studied in the literature for which the value of the packing measure was known. Next, we are going to analyze these same classes utilizing the point of view provided by Theorem 1. This allows, on the one hand, to obtain automatically estimates for the results conjectured in [14] and, on the other hand, to test the effectiveness of the algorithm when the exact value of the packing measure is known. Moreover, we study some self-similar sets $E$ for which, although the value of $P^{s}(E)$ is unknown, a conjecture can be made. In these cases, the algorithm's output provides, for every $k \leq k_{\max }$, an estimate $\tilde{\mathbf{M}}_{k}$ of the value of $P^{s}(E)$ and $100 \%$ confidence intervals $I_{k}:=\left[\tilde{M}_{k}-\varepsilon_{k}, \tilde{M}_{k}+\varepsilon_{k}\right]$ (see Theorem 1). This fact allows us to reject the hypothesis $\alpha=P^{s}(E)$ when $\alpha \notin I_{k}$. If $\alpha \in I_{k}$, then the hypothesis cannot be ruled out as $\left|P^{s}(E)-\alpha\right| \leq 2 \varepsilon_{k}$ is guaranteed. Here $k_{\max }$ denotes the iteration on which the algorithm's run was stopped.

The results presented in Tables (1), (2), (3), and (4), include, for completeness, the values of the constants $s, q_{k_{\max }}$, $Q$, and $\varepsilon_{k_{\max }}$ involved in Theorem 1. We note that, although all the computations have been made using double precision arithmetic, the number of decimal places displayed for the values of $Q, \varepsilon_{k_{\max }}$, and $\tilde{\mathbf{M}}_{k_{\max }}$ has been reduced in order to simplify the presentation

An interesting feature of the algorithm is that in some cases the output stabilizes at an early iteration. The parameter $k_{s t b}$ has been included in the tables to indicate the iteration at which the algorithm output stabilizes in the sense that, for all $k \in\left[k_{s t b}, k_{\text {max }}\right] \cap \mathbb{N}^{+}$, there holds $\tilde{M}_{k}=\tilde{M}_{k_{s t b}}$, after rounding to 14 decimal places.

The computer codes were written in Fortran 90. They were run on the HPC of the Complutense University of Madrid (see www.campusmoncloa.es/es/infraestructuras/eolo for technical description).

\section{Cantor sets in the real line}

Let $C_{r}$ be the linear Cantor set obtained as the attractor of the iterated function system

$$
\left\{f_{1}(x)=r x, \quad f_{2}(x)=1-r+r x\right\}, \quad x \in[0,1] \text { and } 0<r<\frac{1}{2} .
$$

We know by [6] that, for all $r \in\left(0, \frac{1}{2}\right)$,

$$
P^{s}\left(C_{r}\right)=\left(2 \frac{1-r}{r}\right)^{s}
$$

where $s=-\frac{\log 2}{\log r}$ is the similarity dimension of $C_{r}$. Moreover, Algorithm 5 was implemented in [14] for the family $C_{r}$, yielding outputs that coincide with the corresponding values given by (25) (see $\tilde{M}_{20}$ in Table 1). We applied 


\begin{tabular}{|c|c|c|c|c|c|c|}
\hline $\mathbf{r}$ & $\mathbf{s}$ & $\mathbf{q}_{\mathbf{2 0}}$ & $\mathbf{Q}$ & $\tilde{\mathbf{M}}_{\mathbf{2 0}}=\mathbf{P}^{\mathbf{s}}\left(\mathbf{C}_{\mathbf{r}}\right)$ & $\varepsilon_{\mathbf{2 0}}$ & $\mathbf{k}_{\text {stb }}$ \\
\hline $1 / 4$ & $\frac{\log 2}{\log 4}$ & 1 & 1.41421 & 2.449489742783 & $3.63798 \times 10^{-12}$ & 2 \\
\hline $1 / 3$ & $\frac{\log 2}{\log 3}$ & 1 & 1.50000 & 2.398046289121 & $1.68126 \times 10^{-9}$ & 2 \\
\hline 0.38 & $-\frac{\log 2}{\log 0.38}$ & 2 & 1.49896 & 2.333213028519 & $5.56338 \times 10^{-8}$ & 3 \\
\hline 0.383 & $-\frac{\log 2}{\log 0.383}$ & 2 & 1.49695 & 2.327991242710 & $6.58217 \times 10^{-8}$ & 3 \\
\hline 0.45 & $-\frac{\log 2}{\log 0.45}$ & 3 & 1.35502 & 2.172506324847 & $3.98266 \times 10^{-6}$ & 8 \\
\hline
\end{tabular}

Table 1: Linear Cantor sets $C_{r}$.

(4) to the class $C_{r}$ in order to check the effectiveness of the bounds given by Theorem 1 . The results for the final iteration $k_{\max }=20$ of the algorithm are presented in Table 1 .

Observe that for these examples the accuracies of the values of $P^{s}\left(C_{r}\right)$ vary from ten to four decimal places (in the worse case):

$$
\begin{aligned}
\mathbf{2 . 4 4 9 4 8 9 7 4 2 7 7 9} & \leq P^{s}\left(C_{0.25}\right) \leq \mathbf{2 . 4 4 9 4 8 9 7 4 2 7 8 7} \\
\mathbf{2 . 3 9 8 0 4 6 2} 87 & \leq P^{s}\left(C_{1 / 3}\right) \leq \mathbf{2 . 3 9 8 0 4 6 2 9 1} \\
\mathbf{2 . 3 3 3 2 1 2 9} & \leq P^{s}\left(C_{0.38}\right) \leq \mathbf{2 . 3 3 3 2 1 3 1} \\
\mathbf{2 . 3 2 7 9 9 1 1 7} & \leq P^{s}\left(C_{0.383}\right) \leq \mathbf{2 . 3 2 7 9 9 1 3 1} \\
\mathbf{2 . 1 7 2 5} 02 & \leq P^{s}\left(C_{0.45}\right) \leq \mathbf{2 . 1 7 2 5 1 1}
\end{aligned}
$$

\section{Sierpinski gaskets}

Let $S_{r}$ be the self-similar set associated to the system $\Psi=\left\{f_{1,} f_{2}, f_{3}\right\}$ where

$$
\begin{aligned}
& f_{1}(\mathbf{x})=r \mathbf{x} \\
& f_{2}(\mathbf{x})=r \mathbf{x}+(1-r, 0) \\
& f_{3}(\mathbf{x})=r \mathbf{x}+(1-r)\left(\frac{1}{2}, \frac{\sqrt{3}}{2}\right)
\end{aligned}
$$

for $r \in(0,1)$ and $\mathbf{x} \in \mathbb{R}^{2}$. If $r \in\left(0, \frac{1}{2}\right)$, then $S_{r}$ is a Sierpinski gasket of similarity dimension $s=-\frac{\log 3}{\log r}$ satisfying the SSC.

By [14] we know that, for all $r \in\left(0, \frac{1}{3}\right]$,

$$
P^{s}\left(S_{r}\right)=g_{1}(r), \quad \text { where } g_{1}(r):=\left(2 \frac{1-r}{r}\right)^{s} .
$$

The results presented in Table 2 show that Theorem 1 in combination with Algorithm 5 provides quite complete information on the packing measure of the family $\left\{S_{r}\right\}_{r \in\left(0, \frac{1}{2}\right)}$ : When $r \in\left(0, \frac{1}{3}\right]$, Algorithm 5 recovers the value of $P^{s}\left(S_{r}\right)$, and when $r>\frac{1}{3}$, Theorem 1 provides an approximate value for $P^{s}\left(S_{r}\right)$.

In order to analyze the behavior of the family $S_{r}$ with respect to (27), Table 2 is divided into three cases: $r \leq 1 / 3$, where (27) holds; $r>\frac{1}{3}$ and $g_{1}(r) \in I_{k_{\max }}$, where the supposition (27) cannot be rejected as $\left|P^{s}(E)-g_{1}(r)\right| \leq 2 \varepsilon_{k_{\max }}$ is guaranteed; and $r$ satisfying $g_{1}(r) \notin I_{k_{\max }}$, where (27) can be ruled out. For completeness, Table 2 includes the values of $g_{1}(r)$ as well.

The packing measure bounds obtained from applying Theorem 1 to the preceding examples are: 


\begin{tabular}{|c|c|c|c|c|c|c|c|c|}
\hline $\mathbf{r} \leq \frac{1}{3}$ & $\mathbf{s} \leq \mathbf{1}$ & $\mathbf{k}_{\max }$ & $\mathbf{q}_{\mathbf{k}_{\max }}$ & $\mathbf{Q}$ & $\tilde{\mathbf{M}}_{\mathbf{k}_{\max }}=\mathbf{P}^{\mathbf{s}}\left(\mathbf{K}_{\mathbf{r}}\right)=\mathbf{g}_{\mathbf{1}}(\mathbf{r})$ & $\varepsilon_{\mathbf{k}_{\max }}$ & $\mathbf{g}_{\mathbf{1}}(\mathbf{r})$ & $\mathbf{k}_{\mathbf{s t b}}$ \\
\hline $1 / 27$ & $\frac{\log 3}{\log 27}$ & 10 & 1 & 1.05265 & 3.732511156817 & $1.28830 \times 10^{-14}$ & 3.732511156817 & 2 \\
\hline 0.2 & $-\frac{\log 3}{\log 0.2}$ & 12 & 1 & 1.17602 & 4.134802967588 & $3.16650 \times 10^{-8}$ & 4.134802967588 & 2 \\
\hline $1 / 4$ & $\frac{\log 3}{\log 4}$ & 12 & 1 & 1.15470 & 4.136854781603 & $5.66827 \times 10^{-7}$ & 4.136854781603 & 2 \\
\hline 0.33 & $-\frac{\log 3}{\log 0.33}$ & 12 & 1 & 1.00983 & 4.009348546810 & $1.99027 \times 10^{-5}$ & 4.009348546810 & 3 \\
\hline $1 / 3$ & 1 & 12 & 1 & 1 & 4 & $2.25801 \times 10^{-5}$ & 4 & 2 \\
\hline $\mathbf{r}>\frac{1}{3}$ & $\mathbf{s}>\mathbf{1}$ & & & & $\tilde{\mathbf{M}}_{\mathbf{k}_{\max }}, \mathbf{g}_{\mathbf{1}}(\mathbf{r}) \in \mathbf{I}_{\mathbf{k}_{\max }}$ & & & \\
\hline 0.335 & $-\frac{\log 3}{\log 0.335}$ & 12 & 2 & 0.99993 & 3.995192673194 & $7.24702 \times 10^{-5}$ & 3.995192673194 & 3 \\
\hline 0.36 & $-\frac{\log 3}{\log 0.36}$ & 12 & 2 & 0.98125 & 3.912076663518 & $1.89640 \times 10^{-4}$ & 3.912076663518 & 3 \\
\hline 0.365 & $-\frac{\log 3}{\log 0.365}$ & 12 & 2 & 0.97322 & 3.892897543783 & $2.27289 \times 10^{-4}$ & 3.892890309768 & 3 \\
\hline 0.37 & $-\frac{\log 3}{\log 0.37}$ & 12 & 2 & 0.96364 & 3.872834140179 & $2.71384 \times 10^{-4}$ & 3.872817437454 & $>k_{\max }$ \\
\hline 0.385 & $-\frac{\log 3}{\log 0.385}$ & 12 & 2 & 0.92517 & 3.807311991619 & $4.51402 \times 10^{-4}$ & 3.807142406190 & $>k_{\max }$ \\
\hline & & & & & $\tilde{\mathbf{M}}_{\mathbf{k}_{\max }}, \mathbf{g}_{\mathbf{1}}(\mathbf{r}) \notin \mathbf{I}_{\mathbf{k}_{\max }}$ & & & \\
\hline 0.39 & $-\frac{\log 3}{\log 0.39}$ & 13 & 2 & 0.90895 & 3.783682419751 & $2.06943 \times 10^{-4}$ & 3.783386572225 & $>k_{\max }$ \\
\hline 0.42 & $-\frac{\log 3}{\log 0.42}$ & 15 & 3 & 0.77328 & 3.629197993783 & $2.83972 \times 10^{-4}$ & 3.620358378152 & $>k_{\max }$ \\
\hline
\end{tabular}

Table 2: Sierpinski gaskets $\left\{S_{r}\right\}$

\begin{tabular}{|c|c|c|c|}
\hline $\mathbf{k}_{\max }$ & $\tilde{\mathbf{M}}_{\mathbf{k}_{\max }}$ & $\mathbf{I}_{\mathbf{k}_{\max }}$ & CPU \\
\hline 11 & 3.872849586344 & $(\mathbf{3 . 8 7 2 1 1 6 1 1 , 3 . 8 7 3 5 8 3 0 6 )}$ & 10 minutes \\
\hline 12 & 3.872834140179 & 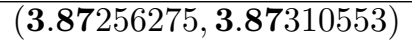 & 92 minutes \\
\hline 13 & 3.872826356688 & $(\mathbf{3 . 8 7 2 7 2 5 9 4 , 3 . 8 7 2 9 2 6 7 7 )}$ & 14 hours and 42 minutes \\
\hline 14 & 3.872821806279 & $(\mathbf{3 . 8 7 2 7 8 4 6 5 , 3 . 8 7 2 8 5 8 9 6 )}$ & 5 days, 23 hours, and 25 minutes \\
\hline 15 & 3.872819763461 & $(\mathbf{3 . 8 7 2 8 0 6 0 1 , 3 . 8 7 2 8 3 3 5 1 )}$ & 59 days \\
\hline
\end{tabular}

Table 3: CPU times for $S_{0.37}$

$$
\begin{aligned}
\mathbf{3 . 7 3 2 5 1 1 1 5 6 8 1 7 2 3 5} & \leq P^{s}\left(S_{\frac{1}{27}}\right) \leq \mathbf{3 . 7 3 2 5 1 1 1 5 6 8 1 7 2 6 2}, \\
\mathbf{4 . 1 3 4 8 0 2 9} 59 & \leq P^{s}\left(S_{\frac{2}{10}}\right) \leq \mathbf{4 . 1 3 4 8 0 2 9 9 9 3}, \\
\mathbf{4 . 1 3 6 8 5 4 2 1} & \leq P^{s}\left(S_{\frac{1}{4}}\right) \leq \mathbf{4 . 1 3 6 8 5 5 3 5} \\
\mathbf{4 . 0 0 9 3 2 8 6} & \leq P^{s}\left(S_{0.33}\right) \leq \mathbf{4 . 0 0 9 3 6 8 5} \\
3.9999774 & \leq P^{s}\left(S_{\frac{1}{3}}\right) \leq 4.0000226 \\
\mathbf{3 . 9 9 5 1 2 0} & \leq P^{s}\left(S_{0.335}\right) \leq \mathbf{3 . 9 9 5 2 6 6} \\
\mathbf{3 . 9 1 1 8 8} & \leq P^{s}\left(S_{0.36}\right) \leq \mathbf{3 . 9 1 2 2 7} \\
\mathbf{3 . 8 9} 267 & \leq P^{s}\left(S_{0.365}\right) \leq \mathbf{3 . 8 9 3 1 3} \\
\mathbf{3 . 8 7 2 5 6} & \leq P^{s}\left(S_{0.37}\right) \leq \mathbf{3 . 8 7 3 1 1} \\
\mathbf{3 . 8 0 6 8 6} & \leq P^{s}\left(S_{0.385}\right) \leq \mathbf{3 . 8 0 7 7 7} \\
\mathbf{3 . 7 8 3 4 7 5} & \leq P^{s}\left(S_{0.39}\right) \leq \mathbf{3 . 7 8 3} 890 \\
\mathbf{3 . 6 2} 891 & \leq P^{s}\left(S_{0.42}\right) \leq \mathbf{3 . 6 2 9 4 9} .
\end{aligned}
$$

In view of Table 2, (27) might also hold for $r$ in some subinterval of $\left[\frac{1}{3}, 0.365\right)$. In particular, when the value of $r$ is one of $0.335,0.36,0.365,0.37$, and 0.385 , the algorithm output approximates the value of $g_{1}(r)$ to $14,15,5,4$, and 3 decimal place accuracy, respectively. However, this is not the case for large $r$, as for $r$ equal to 0.39 or 0.42 , we have that $g_{1}(r) \notin I_{k}$ (see (28), (29) and the values of $g_{1}(0.39)$ and $g_{1}(0.42)$ in Table 2).

Remark 11 Note that to improve the results for larger $r$, a larger value of $k_{\max }$ would be required. However, it is necessary to maintain an equilibrium between the gain in accuracy and the computational time required (see Table 3). The CPU times included in Table 3 are those that were necessary to obtain the values $\tilde{\mathbf{M}}_{k}$ for $k \leq k_{\max }$. Observe that the processing time needed for $k_{\max }=15$ is significantly bigger than that needed when $k_{\max }=13$ (see Section 2.4 for further discussion).

Finally, Figure 1 displays the values of $\tilde{M}_{k}-\varepsilon_{k}, \tilde{M}_{k}, \tilde{M}_{k}+\varepsilon_{k}$, and $g_{1}(r)$ for 34 equidistant values of $r$ in $[0.33,0.45]$. We have used $k=12$ for $r \in[0.33,0.445]$ and $k=13$ for $r \in[0.45,0.495]$. The graphic shows the shape of the 


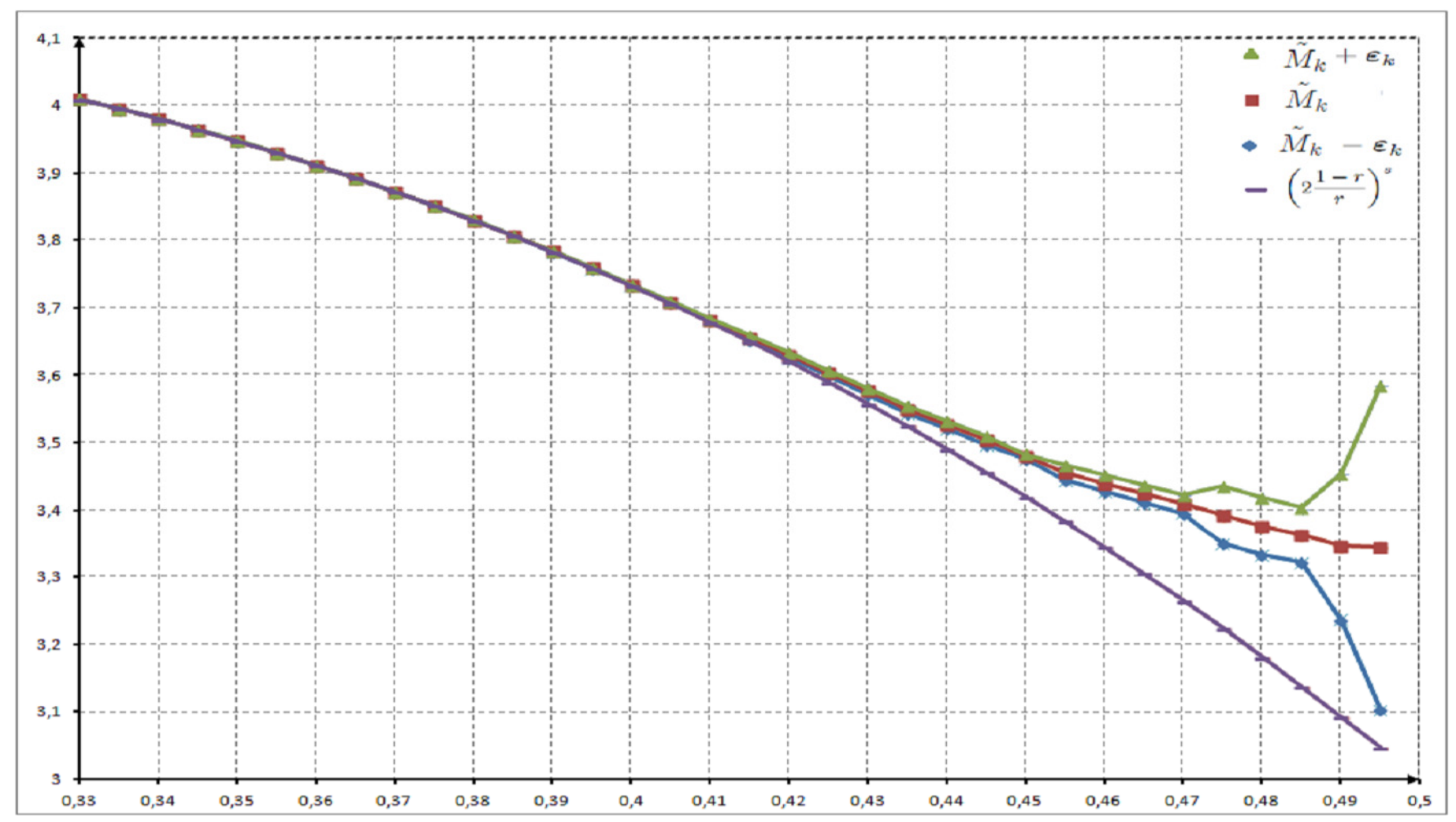

Figure 1: Values of $\tilde{M}_{k}-\varepsilon_{k}, \tilde{M}_{k}, \tilde{M}_{k}+\varepsilon_{k}$, and $g_{1}(r)$ for 34 equidistant values of $r \in[0.33,0.45]$.

curve giving $\tilde{M}_{k}$ as a function of $r$, how the lengths of $I_{k}$ increase with $r$, and also the differences between $\tilde{M}_{k}$ and $g_{1}(r)$ as functions of $r$. It also proves that $g_{1}(r)$ is a lower bound for $P^{s}\left(S_{r}\right)$. This graph provides a computational alternative when the formula $g_{1}(r)$ is not applicable.

\section{Planar Cantor sets}

Let $K_{r}$ be the attractor associated with the iterated function system $\Psi=\left\{f_{1}, f_{2}, f_{3}, f_{4}\right\}$ where

$$
\begin{array}{r}
f_{i}(\mathbf{x})=r \mathbf{x}+b_{i}, \quad i=1,2,3,4, \quad \mathbf{x} \in \mathbb{R}^{2}, \quad 0<r<\frac{1}{2}, \\
b_{1}=(0,0), \quad b_{2}=(1-r, 0), \quad b_{3}=(1-r, 1-r), \quad \text { and } b_{4}=(0,1-r) .
\end{array}
$$

Let $g_{2}(r):=\left(2 \frac{1-r}{r}\right)^{s}$ where $s=-\frac{\log 4}{\log r}$. In [14] it is proved that

$$
P^{s}\left(K_{r}\right)=g_{2}(r)
$$

for every $0<r \leq \frac{1}{4}$, and in [3] the same formula is shown to be true for $\frac{1}{4}<r<\frac{\sqrt{2}}{4}$.

As in the previous example, Table 4 is divided into three cases illustrating the behavior of the family $K_{r}$ with respect to (31). When $0<r \leq \frac{\sqrt{2}}{4}$, in all cases the output coincides at a very early iteration (see $k_{s t b}$ in Table 4 ) with the corresponding value given by (31). For $\frac{\sqrt{2}}{4}<r \leq 0.4$ we observe that $g_{2}(r) \in I_{k_{\max }}$ and thus, although (31) is proved only for $0<r \leq \frac{\sqrt{2}}{4}$, this hypothesis cannot be discarded. In these cases we observe a coincidence between the values given by $\tilde{\mathbf{M}}_{k}$ and $g_{2}(r)$ that varies from 12 to 1 decimal places. This is not the case for $r=0.42$, as $g_{2}(r) \notin I_{k}$ and (31) can be ruled out (see Table 4 and (32)). We can now see the advantage of combining Algorithm 5 and Theorem 1, as we are able to obtain an estimate $\tilde{\mathbf{M}}_{k}$ and a $100 \%$ confidence interval $I_{k}$ for $P^{s}\left(K_{r}\right)$ regardless of the 


\begin{tabular}{|c|c|c|c|c|c|c|c|c|}
\hline $\mathbf{r} \leq \frac{\sqrt{\mathbf{2}}}{\mathbf{4}}$ & $\mathbf{s}$ & $\mathbf{k}_{\max }$ & $\mathbf{q}_{\mathbf{k}_{\max }}$ & $\mathbf{Q}$ & $\tilde{\mathbf{M}}_{\mathbf{k}_{\max }}=\mathbf{P}^{\mathbf{s}}\left(\mathbf{K}_{\mathbf{r}}\right)=\mathbf{g}_{\mathbf{2}}(\mathbf{r})$ & $\varepsilon_{\mathbf{k}_{\max }}$ & $\mathbf{g}_{\mathbf{2}}(\mathbf{r})$ & $\mathbf{k}_{\mathbf{s t b}}$ \\
\hline 0.2 & $-\frac{\log 4}{\log 0.2}$ & 10 & 1 & 1.07339 & 5.996245070706 & $1.94598 \times 10^{-6}$ & 5.996245070706 & 2 \\
\hline $1 / 4$ & 1 & 10 & 1 & 1 & 6 & $2.15792 \times 10^{-5}$ & 6 & 2 \\
\hline 0.3 & $-\frac{\log 4}{\log 0.3}$ & 11 & 2 & 1.04453 & 5.892731803791 & $2.14177 \times 10^{-4}$ & 5.892731803791 & 3 \\
\hline 0.35 & $-\frac{\log 4}{\log 0.35}$ & 12 & 2 & 0.95179 & 5.656172537869 & $4.80038 \times 10^{-4}$ & 5.656172537869 & 4 \\
\hline $\mathbf{r}>\frac{\sqrt{\mathbf{2}}}{\mathbf{4}}$ & & & & & $\tilde{\mathbf{M}}_{\mathbf{k}_{\max }, \mathbf{g}_{\mathbf{2}}(\mathbf{r}) \in \mathbf{I}_{\mathbf{k}_{\max }}}$ & & & \\
\hline 0.36 & $-\frac{\log 4}{\log 0.36}$ & 10 & 2 & 0.91421 & 5.591584024577 & $5.25726 \times 10^{-3}$ & 5.591584024577 & 4 \\
\hline 0.365 & $-\frac{\log 4}{\log 0.365}$ & 10 & 2 & 0.89297 & 5.557001901721 & $6.05272 \times 10^{-3}$ & 5.557001901721 & 4 \\
\hline 0.37 & $-\frac{\log 4}{\log 0.37}$ & 10 & 2 & 0.87012 & 5.520873608633 & $6.93986 \times 10^{-3}$ & 5.520869632675 & 4 \\
\hline 0.39 & $-\frac{\log 4}{\log 0.39}$ & 11 & 2 & 0.76309 & 5.361914850770 & $4.47849 \times 10^{-3}$ & 5.360487383353 & $>k_{\max }$ \\
\hline 0.395 & $-\frac{\log 4}{\log 0.395}$ & 11 & 3 & 0.73263 & 5.320007312123 & $2.03399 \times 10^{-2}$ & 5.316346629766 & $>k_{\max }$ \\
\hline 0.4 & $-\frac{\log 4}{\log 0.4}$ & 12 & 3 & 0.70079 & 5.277123200420 & $9.18961 \times 10^{-3}$ & 5.270557940489 & $>k_{\max }$ \\
\hline $\mathbf{r}>\frac{\sqrt{2}}{4}$ & & & & & $\tilde{\mathbf{M}}_{\mathbf{k}_{\max }}, \mathbf{g}_{\mathbf{2}}(\mathbf{r}) \notin \mathbf{I}_{\mathbf{k}_{\max }}$ & & & \\
\hline 0.42 & $-\frac{\log 4}{\log 0.42}$ & 11 & 3 & 0.56150 & 5.12798012945 & $3.52941 \times 10^{-2}$ & 5.070677295108 & $>k_{\max }$ \\
\hline
\end{tabular}

Table 4: Planar Cantor sets $\left\{K_{r}\right\}$

existence of an exact formula (see the estimates below). These examples also show that $g_{2}(r)$ is a lower bound for $P^{s}\left(K_{r}\right)$.

The bounds obtained from applying Theorem 1 to the preceding examples are:

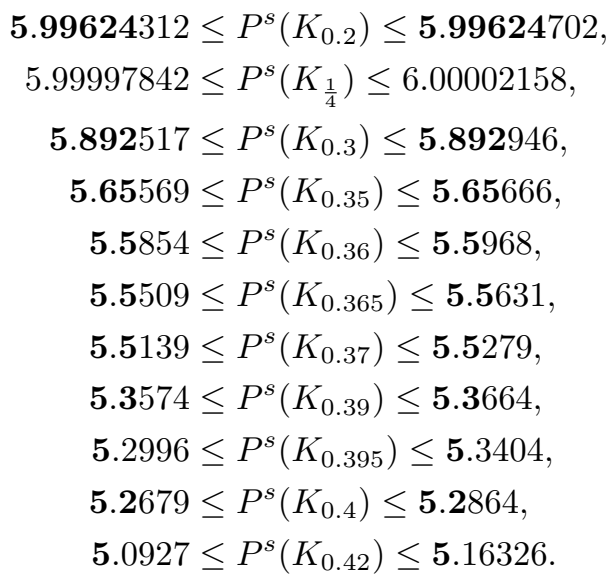

\subsection{Computability of the packing measure: the general case}

A general pattern emerges from the above examples. For self-similar sets with small contraction ratios there exists a formula that gives the exact value of the packing measure, and the optimal density is attained for an optimal ball, which can be found by the algorithm. As the contraction ratios increase, these statements cease to be valid. This raises the problem of whether, for a given case, there exists an optimal ball that can be computed in finite time, and for which the exact value of $P^{s}(E)$ can be computed to arbitrary accuracy. We say that a self-similar set with these properties enjoys the finite time computability property.

Several things must happen for the finite time computability property to hold. The optimal ball $B^{*}$ should be centered in one of the clouds $A_{k}$, and the boundary of the optimal ball should also lie in some $A_{k}$. If these two conditions hold the optimal ball can be found in finite time, but this does not guarantee that the exact value of $P^{s}(E)$ can be computed in finite time, since the process estimating the exact value of $\mu\left(B^{*}\right)$ can be infinite unless $B^{*}$ is a union of a finite number of cylinders of the $k_{0}$ th generation for some $k_{0}$, since then $\mu\left(B^{*}\right)=\mu_{k}\left(B^{*}\right)$ for $k \geq k_{0}$ (Theorem 4.13 in [13] illustrates this point). All these circumstances should be considered as exceptional events, unless there were found some rigorous proof that they must occur. Thus the general case should be considered as noncomputable in finite time.

If the finite time computability property holds in a particular case, a theoretical argument based on geometric properties can give the exact packing measure, but, in the general case, the only approach that can be taken to calculate $P^{s}(E)$ is a computational one.

In order to illustrate the general case we present below Table 5. It records the intermediate results of the algorithm for a unique self-similar set, the Sierpinski gasket $S_{0.42}$. 


\begin{tabular}{|l|c|c|c|c|c|}
\hline $\mathbf{k}$ & $\mathbf{x}$ & $\mathbf{y}$ & $\mathbf{d}$ & $\tilde{\mathbf{M}}_{k}$ & $\mathbf{I}_{k}$ \\
\hline 5 & $(0,0)$ & $(0.25915848,0.02694808)$ & 0.26055578 & 3.67050829 & $(2.00793066,5.33308593)$ \\
\hline 6 & $(0,0)$ & $(0.13486912,0.21096379)$ & 0.25039050 & 3.65830695 & $(2.96002434,4.35658956)$ \\
\hline 7 & $(0,0)$ & $(0.24634452,0.00475364)$ & 0.24639038 & 3.64297340 & $(3.34969470,3.93625210)$ \\
\hline 8 & $(0,0)$ & $(0.24590539,0)$ & 0.24590539 & 3.63389479 & $(3.51071773,3.75707184)$ \\
\hline 9 & $(0,0)$ & $(0.24519182,0.00275711)$ & 0.24520732 & 3.63071511 & $(3.57898075,3.68244948)$ \\
\hline 10 & $(0,0)$ & $(0.24673228,0.00310930)$ & 0.24675187 & 3.62998849 & $(3.60826005,3.65171693)$ \\
\hline 11 & $(0,0)$ & $(0.24671071,0.00411937)$ & 0.24674510 & 3.62949853 & $(3.62037258,3.63862448)$ \\
\hline 12 & $(0,0)$ & $(0.12700823,0.21145014)$ & 0.24666223 & 3.62928853 & $(3.62545563,3.63312143)$ \\
\hline 13 & $(0,0)$ & $(0.24666388,0.00281924)$ & 0.24667999 & 3.62921523 & $(3.62760541,3.63082505)$ \\
\hline 14 & $(0,0)$ & $(0.24663898,0.00411937)$ & 0.24667338 & 3.62921324 & $(3.62853711,3.62988937)$ \\
\hline 15 & $(0,0)$ & $(0.24663671,0.00424755)$ & 0.24667328 & 3.62919799 & $(3.62891402,3.62948197)$ \\
\hline
\end{tabular}

Table 5: Sierpinski gasket $S_{0.42}$

The columns in this table are: the number $k$ of iterations, the center $x$ and endpoint $y$ of the optimal ball at the $k$ th iteration, the radius $d$, the estimate $\tilde{M}_{k}$ for $P^{s}\left(S_{0.42}\right)$ at the $k$ th iteration, and the interval $I_{k}$ to which we can be sure that $P^{s}\left(S_{0.42}\right)$ belongs. For simplicity all the values are rounded to eight decimal places. One can see that, in spite of the stabilization of $x$, the remaining values change from iterate to iterate until the computational time is too big to continue.

Remark 12 The case $r=0.42$ is a good example illustrating the frontiers of computability of the packing measure. We needed $k_{\max }=15$ to obtain only two digits of accuracy in the estimate of $P^{s}\left(S_{0.42}\right)$. It is difficult to increase $k_{\max }$ because the set $A_{15}$ consists of $3^{15}=14348907$ data points, and for this value the computation required more than one month of CPU time.

\section{Centered Hausdorff measure}

The centered Hausdorff measure is a variant of the Hausdorff measure. The main difference between them is the nature of the coverings used in their definitions. In the case of the centered Hausdorff measure the set of coverings is restricted to closed balls centered at points in the given set (see, e.g. [24], for the standard definition and properties). However, here, instead of the standard definition of $C^{s}$ we use the following relation proved in [11] for totally disconnected self-similar sets:

$$
C^{s}(E)=\min \{\bar{h}(x, d): x \in E \text { and } c \leq d \leq R\}
$$

(see Section 1 for the notational conventions). This can be improved to

$$
C^{s}(E)=\min \{\bar{h}(x, d):(x, d) \in \mathcal{A}\},
$$

with

$$
\mathcal{A}=\left\{(x, d) \in E \times[c, R]: \bar{B}(x, d) \cap E_{j} \neq \emptyset \text { for some } j \in M \text { with } j \neq i_{1}^{x}\right\} .
$$

This is so because, as argued in [11, Remark 6], any ball $B(x, d)$ with $B(x, d) \cap E \subset E_{i_{1}^{x}}$ can be enlarged to a ball $B\left(f_{i_{1}^{x}}^{-1}(x), \frac{d}{r_{i_{1}^{x}}^{x}}\right)$ with $\bar{h}(x, d)=\bar{h}\left(f_{i_{1}^{x}}^{-1}(x), \frac{d}{r_{i_{1}^{x}}}\right)$. The inequality $d<c$ ensures that $B(x, d) \cap E \subset E_{i_{1}^{x}}$ holds true, but, even if $d \geq c$, it might happen that $B(x, d)$ could still be enlarged. The condition used in the above definition of $\mathcal{A}$ rules out, however, any further enlargement of $B(x, d)$.

Similarly to the packing measure case, (34) allows the construction of an algorithm converging to the value of $C^{s}(E)$ through an approximation of the minimal value of $\bar{h}(x, d):=\frac{(2 d)^{s}}{\mu(\bar{B}(x, d))}$. Observe that we are taking closed balls in the definition of $\bar{h}(x, d)$ instead of the open ones used in the packing measure case. Nevertheless, replacing open balls with closed balls in (33) does not make any difference in the limit as, by [15], we know that $\mu(\partial B(x, d))=0$. Moreover, using closed balls has proved to be computationally more efficient.

\subsection{Previous results: centered Hausdorff measure algorithm}

This section describes an improved version of the algorithm developed in [13] for computing the centered Hausdorff measure of totally disconnected self-similar sets. This new version has two novelties. On the one hand, it allows a reduction of the number of calculations needed on each step, making the algorithm faster at the expense of using more memory by caching part of the calculations made in prior iterations instead of recalculating them on each iteration. On the other hand, it uses the more efficient condition (34) in place of (33). 
As the structure of Algorithm 13 is very similar to that of the algorithm for the packing measure, we start the description supposing that $A_{k}, \Delta_{k}$ and $\mu_{k}$ have already been constructed, so that we can see the differences between the two algorithms.

Algorithm 13 (Centered Hausdorff measure) Input of the Algorithm: The system of contracting similitudes and the iteration $k_{\max }$ on which the algorithm's run is stopped.

We begin the description of the algorithm with step 4 as the construction of $A_{k}, \mu_{k}$, and the list of distances is the same as in the packing measure case. Thus, assume steps 1, 2, and 3 are as in Algorithm 5 , and let $k \in \mathbb{N}^{+}$such that $k \leq k_{\max }$.

\section{Construction of $\tilde{\mathbf{m}}_{k}$.}

Given $x \in A_{k}$ :

4.1 Rank in increasing order those distances $d \in \Delta_{k}$ that contain the letter $\mathbf{i}_{k}^{x}$ in their addresses (see (13) for the notation).

4.2 Let $0=d_{1}^{x} \leq d_{2}^{x} \leq \cdots \leq d_{m^{k}}^{x}$ be the list of ordered distances and, for every $j \in\left\{1, \ldots, m^{k}\right\}$, let $t_{j} \in \mathbb{N}$ be such that $d_{j}^{x}=\cdots=d_{j+t_{j}}^{x} \neq d_{j+t_{j}+1}^{x}$. Then,

$$
\mu_{k}\left(\bar{B}\left(x, d_{j}^{x}\right)\right):=\sum_{q=1}^{j+t_{j}} r_{\mathbf{i}_{k}^{x_{q}}}^{s_{q}}
$$

where $x_{q} \in A_{k}$ is the point chosen for calculating the distance $d_{q}^{x}=d\left(x, x_{q}\right), q=1, \ldots, j+t_{j}$.

Observe that, in the particular case when $r_{i}=r$ for all $i \in M$, we have

$$
\mu_{k}\left(B\left(x, d_{j}^{x}\right)\right)=\frac{j+t_{j}}{m^{k}} .
$$

4.3 Compute

$$
\bar{h}_{k}\left(x, d_{j}^{x}\right):=\frac{\left(2 d_{j}^{x}\right)^{s}}{\mu_{k}\left(\bar{B}\left(x, d_{j}^{x}\right)\right)}=\frac{\left(2 d_{j}^{x}\right)^{s}}{\sum_{q=1}^{j+t_{j}} r_{\mathbf{i}_{k}^{x q}}^{s}}
$$

only for those distances $d_{j}^{x}$ in the list satisfying $j_{0}^{x} \leq j \leq m^{k}$, where

$$
j_{0}^{x}=\min \left\{j \in\left\{1, \ldots, m^{k}\right\}: d_{j}^{x}=|x-y| \text { for some } y \in A_{k} \text { with } i_{1}^{x} \neq i_{1}^{y}\right\},
$$

according to (34) and (35).

Henceforth we use the following notation. Given $k \in \mathbb{N}^{+}$and $x \in A_{k}$, we define

$$
\bar{D}_{k}^{x}:=\cup_{j=j_{0}^{x}}^{m^{k}} d_{j}^{x}
$$

and

$$
\bar{D}_{k}:=\cup_{x \in A_{k}} \bar{D}_{k}^{x}
$$

Observe that $\bar{D}_{k}$ takes values only within the interval $[c, R]$.

4.4 Find the minimum

$$
\bar{m}_{k}(x)=\min \left\{\bar{h}_{k}\left(x, d_{j}^{x}\right): j=j_{0}, \ldots, m^{k}\right\}
$$

of the values computed in step 4.3.

5 Repeat step 4 for each $x \in A_{k}$.

6 Take the minimum

$$
\tilde{m}_{k}:=\min \left\{\bar{m}_{k}(x): x \in A_{k}\right\}
$$

of the $m^{k}$ values computed in step 5 . Note that

$$
\tilde{m}_{k}:=\bar{h}_{k}\left(\tilde{x}_{k}, \tilde{d}_{k}\right):=\frac{\left(2 \tilde{d}_{k}\right)^{s}}{\mu_{k}\left(\bar{B}\left(\tilde{x}_{k}, \tilde{d}_{k}\right)\right)}=\min _{x \in A_{k}} \min _{d \in \bar{D}_{k}^{x}} \bar{h}_{k}(x, d) .
$$

\section{Remark 14}

1. Note that, by (38), for any $x \in A_{k}$,

$$
\begin{aligned}
d \in \quad & \bar{D}_{k}^{x} \Longleftrightarrow d=|x-y| \text { for some } y \in A_{k} \text { and } \\
& \text { there exists } z \in A_{k} \cap \bar{B}(x, d) \text { with } i_{1}^{z} \neq i_{1}^{x} .
\end{aligned}
$$

2. Let $q \in \mathbb{N}^{+}$be such that $R r_{\max }^{q} \leq c<R r_{\max }^{q-1}$. Then, for any $k \in \mathbb{N}^{+}$and $(x, d) \in A_{k} \times \bar{D}_{k}^{x}$, there holds $E_{\mathbf{i}_{q}^{x}} \subset \bar{B}(x, d)$, whence

$$
\mu_{k}(\bar{B}(x, d)) \geq \mu\left(E_{\mathbf{i}_{q}^{x}}\right) \geq r_{\min }^{q s}
$$




\subsection{Rate of convergence of the centered Hausdorff measure algorithm}

This section is devoted to showing the rate of convergence of Algorithm 13. Since the proof of Theorem 2 does not use the convergence $C^{s}(E)=\lim _{k \rightarrow \infty} \tilde{m}_{k}$, this gives an alternative proof of the convergence of Algorithm 13 .

As in Section 2.2, we show first that the construction of appropriate approximating balls allows a comparison between the densities given in (34) with those computed by Algorithm 13. The proof of Theorem 2 is postponed to the end of the section.

Lemma 15 Given $k \in \mathbb{N}^{+}$and $(x, d) \in \mathcal{A}$ there exists $\left(x^{\prime}, d^{\prime}\right) \in A_{k} \times \bar{D}_{k}^{x^{\prime}}$ such that

(i) $\left|x-x^{\prime}\right| \leq R r_{\max }^{k}$,

(ii) $d^{\prime} \leq d+2 R r_{\max }^{k}$,

(iii) $\mu(\bar{B}(x, d)) \leq \mu_{k}\left(\bar{B}\left(x^{\prime}, d^{\prime}\right)\right)$.

Proof. Let $k \in \mathbb{N}^{+}$and $(x, d) \in \mathcal{A}$ (see (35) for the notation). Take the unique point $x^{\prime} \in A_{k}$ such that $E_{\mathbf{i}_{k}^{x}}=E_{\mathbf{i}_{k}^{x^{\prime}}}$. Then (i) holds. Now set $L:=\left\{y \in A_{k}: E_{\mathbf{i}_{k}^{y}} \cap \bar{B}(x, d) \neq \emptyset\right\}$ and $d^{\prime}:=\max \left\{\left|y-x^{\prime}\right|: y \in L\right\}$.

Observe that, by definition of $\mathcal{A}$, there exists $\mathbf{j}_{k} \in M^{k}$ with $j_{1} \neq i_{1}^{x}$ and $\bar{B}(x, d) \cap E_{\mathbf{j}_{k}} \neq \emptyset$. Moreover, taking $z \in A_{k}$ such that $E_{\mathbf{i}_{k}^{z}}=E_{\mathbf{j}_{k}}$, we obtain that $z \in L$ whence $\left|z-x^{\prime}\right| \leq d^{\prime}$. This, in turn, implies that $d^{\prime} \in \bar{D}_{k}^{x^{\prime}}$ because $z \in \bar{B}\left(x^{\prime}, d^{\prime}\right) \cap A_{k}$ and $i_{1}^{x^{\prime}}=i_{1}^{x} \neq j_{1}=i_{1}^{z}($ see $(41))$.

The proof of (ii) follows from the triangle inequality, taking $t^{\prime} \in L \cap \partial \bar{B}\left(x^{\prime}, d^{\prime}\right)$ and $t \in E_{\mathbf{i}_{k}^{\prime}} \cap \bar{B}(x, d)$ :

$$
d^{\prime}=\left|x^{\prime}-t^{\prime}\right| \leq\left|x^{\prime}-x\right|+|x-t|+\left|t-t^{\prime}\right| \leq d+2 R r_{\max }^{k} .
$$

Finally, (iii) holds because $L \subset \bar{B}\left(x^{\prime}, d^{\prime}\right) \cap A_{k}$ and hence

$$
\mu(\bar{B}(x, d)) \leq \mu\left(\cup_{\mathbf{i}_{k} \in M^{k}}\left\{E_{\mathbf{i}_{k}}: E_{\mathbf{i}_{k}} \cap \bar{B}(x, d) \neq \emptyset\right\}\right)=\mu_{k}(L) \leq \mu_{k}\left(\bar{B}\left(x^{\prime}, d^{\prime}\right)\right) .
$$

Lemma 16 Given $(x, d) \in A_{k} \times \bar{D}_{k}^{x}$, there exists $d^{\prime} \in[c, R]$ with $\left(x, d^{\prime}\right) \in \mathcal{A}$, and such that

(i) $d^{\prime} \leq d+R r_{\max }^{k}$ and

(ii) $\mu_{k}(\bar{B}(x, d)) \leq \mu\left(\bar{B}\left(x, d^{\prime}\right)\right)$.

Proof. Let $P:=\left\{\mathbf{i}_{k} \in M^{k}: E_{\mathbf{i}_{k}} \cap \bar{B}(x, d) \neq \emptyset\right\}$ and $L:=\bigcup_{\mathbf{i}_{k} \in P} E_{\mathbf{i}_{k}}$. Set $d^{\prime}:=\max \{|y-x|: y \in L\}$. By definition $L \subset \bar{B}\left(x, d^{\prime}\right) \cap E$. Thus

$$
\mu_{k}(\bar{B}(x, d)) \leq \mu_{k}\left(\left\{y \in A_{k}: E_{\mathbf{i}_{k}^{y}} \cap \bar{B}(x, d) \neq \emptyset\right\}\right)=\mu(L) \leq \mu\left(\bar{B}\left(x, d^{\prime}\right)\right)
$$

which proves (ii). The proof of (i) follows by taking $y \in L \cap \partial \bar{B}\left(x, d^{\prime}\right)$ and $z \in E_{\mathbf{i}_{k}^{y}} \cap \bar{B}(x, d)$ and applying the triangle inequality:

$$
d^{\prime}=|x-y| \leq|x-z|+|z-y| \leq d+R r_{\max }^{k} .
$$

Finally, (41) implies the existence of $z \in A_{k} \cap \bar{B}(x, d)$ with $i_{1}^{z} \neq i_{1}^{x}$. This proves $\left(x, d^{\prime}\right) \in \mathcal{A}$ since $E_{\mathbf{i}_{k}^{z}} \in L$ and $B\left(x, d^{\prime}\right) \cap E_{\mathbf{i}_{\mathbf{k}}} \neq \emptyset$

We are now ready to prove our main result for the centered Hausdorff measure.

Proof of Theorem 2. Suppose first that $\tilde{m}_{k} \geq C^{s}(E)$. Let $(x, d) \in \mathcal{A}$ be such that $C^{s}(E)=\frac{(2 d)^{s}}{\mu(\bar{B}(x, d))}($ see $(34))$ and take $\left(x^{\prime}, d^{\prime}\right) \in A_{k} \times \bar{D}_{k}^{x^{\prime}}$ as in Lemma 15. Then (40), (ii) and (iii) of Lemma 15, (21), and the mean value theorem imply

$$
\begin{aligned}
\tilde{m}_{k}-C^{s}(E) & \leq \frac{\left(2 d^{\prime}\right)^{s}}{\mu_{k}\left(\bar{B}\left(x^{\prime}, d^{\prime}\right)\right)}-\frac{(2 d)^{s}}{\mu(\bar{B}(x, d))} \\
& \leq 2^{s} \frac{\left(d^{\prime}\right)^{s}-d^{s}}{\mu_{k}\left(\bar{B}\left(x^{\prime}, d^{\prime}\right)\right)} \leq \frac{s 2^{s+1} Q}{r_{\min }^{q s}} R r_{\max }^{k},
\end{aligned}
$$

where $Q$ is as in (7).

Finally, if $\tilde{m}_{k} \leq C^{s}(E),(40)$, the mean value theorem, and Lemma 16 with $(x, d) \in A_{k} \times \bar{D}_{k}^{x}$ such that $\tilde{m}_{k}=\frac{(2 d)^{s}}{\mu_{k}(\bar{B}(x, d))}$, imply

$$
\begin{gathered}
C^{s}(E)-\tilde{m}_{k} \leq \frac{\left(2 d^{\prime}\right)^{s}}{\mu\left(\bar{B}\left(x, d^{\prime}\right)\right)}-\frac{(2 d)^{s}}{\mu_{k}(\bar{B}(x, d))} \\
\leq 2^{s} \frac{\left(d^{\prime}\right)^{s}-d^{s}}{\mu_{k}\left(\bar{B}\left(x, d^{\prime}\right)\right)} \leq s 2^{s} \mathcal{Q} R \frac{r_{\max }^{k}}{r_{\min }^{q s}},
\end{gathered}
$$

where $Q$ is as in (7) and $d^{\prime}$ is given by Lemma 16 . 


\begin{tabular}{|c|c|c|c|c|c|c|c|c|}
\hline $\mathbf{r} \leq \frac{1}{3}$ & $\mathbf{q}$ & $\mathbf{s}$ & $\mathbf{k}_{\max }$ & $\mathcal{Q}$ & $\tilde{\mathbf{m}}_{\mathbf{k}_{\max }}=\mathbf{C}^{\mathbf{s}}\left(\mathbf{C}_{\mathbf{r}}\right)=\mathbf{g}_{\mathbf{3}}(\mathbf{r})$ & $\epsilon_{\mathbf{k}_{\max }}$ & $\mathbf{g}_{\mathbf{3}}(\mathbf{r})$ & $\mathbf{k}_{\mathbf{s t b}}$ \\
\hline$\frac{1}{4}$ & 1 & $\frac{\log 2}{\log 4}$ & 20 & 1.41421 & 1.224744871392 & $3.63798 \times 10^{-12}$ & 1.224744871392 & 2 \\
\hline$\frac{1}{3}$ & 1 & $\frac{\log 2}{\log 3}$ & 20 & 1.5 & 1.199023144561 & $1.68126 \times 10^{-9}$ & 1.199023144561 & 3 \\
\hline $\mathbf{r}>\frac{1}{3}$ & & & & & $\tilde{\mathbf{m}}_{\mathbf{k}_{\max }}, \mathbf{g}_{\mathbf{3}}(\mathbf{r}) \in \mathbf{I}_{\mathbf{k}_{\max }}$ & & & \\
\hline 0.351 & 2 & $-\frac{\log 2}{\log 0.351}$ & 20 & 1.50552 & 1.188484857299 & $1.01650 \times 10^{-8}$ & 1.188484857299 & 4 \\
\hline 0.3518 & 2 & $-\frac{\log 2}{\log 0.3518}$ & 20 & 1.50562 & 1.187959585122 & $1.06731 \times 10^{-8}$ & 1.187959585122 & 4 \\
\hline & & & & & $\tilde{\mathbf{m}}_{k_{\max }}, g_{3}(r) \notin I_{k_{\max }}$ & & & \\
\hline 0.3519 & 2 & $-\frac{\log 2}{\log 0.3519}$ & 20 & 1.50563 & 1.187703097489 & $1.07383 \times 10^{-8}$ & 1.187893625780 & 4 \\
\hline 0.4 & 2 & $-\frac{\log 2}{\log 0.4}$ & 20 & 1.47986 & 1.084545262462 & $1.66350 \times 10^{-7}$ & 1.147884787390 & 7 \\
\hline 0.45 & 3 & $-\frac{\log 2}{\log 0.45}$ & 21 & 1.35502 & 1.031518332488 & $1.79220 \times 10^{-6}$ & 1.086253162423 & 19 \\
\hline
\end{tabular}

Table 6: Linear Cantor sets $\left\{C_{r}\right\}$

\subsection{Examples}

As in Section 2.3, we now analyze the examples studied in [13] taking into account Theorem 2. We observe that Theorem 2 gives an automated tool for proving the conjectures on the values of $C^{s}$ given in [13]. Let $I_{k}$ be the closed interval $I_{k}:=\left[\tilde{m}_{k}-\epsilon_{k}, \tilde{m}_{k}+\epsilon_{k}\right]$ where $\tilde{m}_{k}$ and $\epsilon_{k}$ are defined in Theorem 2.

\section{Cantor type sets in the real line.}

Let $\left\{C_{r}\right\}_{r \in\left(0, \frac{1}{2}\right)}$ be the family of linear Cantor set defined by (24). In [26] it is proved that if $0<r \leq \frac{1}{3}$, then

$$
C^{s}\left(C_{r}\right)=g_{3}(r) \text { where } g_{3}(r):=2^{s}(1-r)^{s},
$$

and $s=-\frac{\log 2}{\log r}$ is the similarity dimension of $\left\{C_{r}\right\}$.

Table 1 records the results obtained from applying Theorem 2 in combination with Algorithm 13 to the family $\left\{C_{r}\right\}$. As in Section 2.3, the examples are chosen to illustrate the behavior of $C^{s}\left(C_{r}\right)$ with respect to (43).

As a consequence of these results we obtain the bounds:

$$
\begin{aligned}
1.224744871387 & <C^{s}\left(C_{\frac{1}{4}}\right)<\mathbf{1 . 2 2 4 7 4 4 8 7 1 3 9 6}, \\
1.1990231428 & <C^{s}\left(C_{\frac{1}{3}}\right)<\mathbf{1 . 1 9 9 0 2 3 1 4 6 3 ,} \\
1.188484847 & <C^{s}\left(C_{0.351}\right)<\mathbf{1 . 1 8 8 4 8 4 8 6 8}, \\
1.187959574 & <C^{s}\left(C_{0.3518}\right)<\mathbf{1 . 1 8 7 9 5 9 5 9 6 ,} \\
1.18770308 & <C^{s}\left(C_{0.3519}\right)<\mathbf{1 . 1 8 7 7 0 3} 11, \\
1.08454509 & <C^{s}\left(C_{\frac{4}{10}}\right)<\mathbf{1 . 0 8 4 5 4 5 4 3}, \\
1.031516 & <C^{s}\left(C_{0.45}\right)<\mathbf{1 . 0 3 1 5 2 1}
\end{aligned}
$$

The algorithm recovers the value given by (43) in the cases where $r \leq 1 / 3$. To our knowledge, there is no general formula for $C^{s}\left(C_{r}\right)$ when $r>\frac{1}{3}$, but in these cases we obtain estimates of its value to accuracies of 7,6 , and 4 decimal places, namely, $C^{s}\left(C_{0.351}\right) \simeq 1.1884848, C^{s}\left(C_{0.3518}\right) \simeq 1.1879595, C^{s}\left(C_{0.3519}\right) \simeq 1.187703, C^{s}\left(C_{\frac{4}{10}}\right) \simeq 1.084545$, and $C^{s}\left(C_{0.45}\right) \simeq 1.0315$. Moreover, as in Section 2.3, (45), (46), and (47) show that (43) is, in general, not valid when $r>\frac{1}{3}$. In fact, $g_{3}(r)$ is an upper bound for $C^{s}\left(C_{r}\right)$.

2. Sierpinski gaskets. Let $S_{r}$ be the class of Sierpinski gaskets defined by (26) and let

$$
g_{4}(r):=\left[2(1-r)\left(r^{2}+r+1\right)^{\frac{1}{2}}\right]^{s}
$$

where $s=-\frac{\log (3)}{\log (r)}$. The results obtained in [13] for this class of Sierpinski gaskets led to the conjecture

$$
C^{s}\left(S_{r}\right)=g_{4}(r) \quad \text { for all } r<0.25 \text {. }
$$

In Table 7 we can see that, even when (48) was conjectured for $r<0.25$, the algorithm output approximates the value given by (48) in all the cases where $r \leq 0.277$ (with accuracy of more than 12 decimal places). For the cases where $r \geq 0.278$ we observe the opposite behavior, $g_{4}(r) \notin I_{k_{\max }}$, so (48) cannot hold, and $g_{4}(r)$ is an upper bound for $C^{s}\left(S_{r}\right)$. 


\begin{tabular}{|c|c|c|c|c|c|c|c|c|}
\hline $\mathbf{r}$ & $\mathbf{s}$ & $\mathbf{q}$ & $\mathbf{k}_{\max }$ & $\mathcal{Q}$ & $\tilde{\mathbf{m}}_{\mathbf{k}_{\max }}, \mathbf{g}_{\mathbf{4}}(\mathbf{r}) \in I_{\mathbf{k}_{\max }}$ & $\epsilon_{\mathbf{k}_{\max }}$ & $\mathbf{g}_{\mathbf{4}}(\mathbf{r})$ & $\mathbf{k}_{\mathbf{s t b}}$ \\
\hline $1 / 27$ & $\frac{\log 3}{\log 27}$ & 1 & 10 & 1.05265 & 1.252010347930 & $1.28830 \times 10^{-14}$ & 1.252010347930 & 3 \\
\hline 0.2 & $-\frac{\log 3}{\log 0.2}$ & 1 & 12 & 1.17602 & 1.483264747602 & $3.16650 \times 10^{-8}$ & 1.483264747602 & 3 \\
\hline $1 / 4$ & $\frac{\log 3}{\log 4}$ & 1 & 12 & 1.15470 & 1.535835728296 & $5.66827 \times 10^{-7}$ & 1.535835728296 & 3 \\
\hline 0.277 & $-\frac{\log 3}{\log 0.277}$ & 1 & 12 & 1.12349 & 1.560819225967 & $2.13042 \times 10^{-6}$ & 1.560819225967 & $>k_{\max }$ \\
\hline & & & & & $\tilde{\mathbf{m}}_{\mathbf{k}_{\max }, \mathbf{g}_{4}(\mathbf{r}) \notin \mathbf{I}_{\mathbf{k}_{\max }}}$ & & & \\
\hline 0.278 & $-\frac{\log 3}{\log 0.278}$ & 1 & 12 & 1.12202 & 1.561597393347 & $2.23163 \times 10^{-6}$ & 1.561690520340 & 5 \\
\hline $1 / 3$ & 1 & 1 & 13 & 1 & 1.543702825201 & $7.52671 \times 10^{-6}$ & 1.602467233540 & $>k_{\max }$ \\
\hline 0.4 & $-\frac{\log 3}{\log 0.4}$ & 2 & 12 & 1 & 1.472023977311 & $8.31250 \times 10^{-4}$ & 1.624473448850 & $>k_{\max }$ \\
\hline
\end{tabular}

Table 7: Sierpinski gaskets $\left\{S_{r}\right\}$

Next we give the bounds provided by Theorem 2:

$$
\begin{aligned}
1.25201034793033 & <C^{s}\left(S_{\frac{1}{27}}\right)<\mathbf{1 . 2 5 2 0 1 0 3 4 7 9 3 0 3 5}, \\
1.483264715 & <C^{s}\left(S_{\frac{2}{10}}\right)<\mathbf{1 . 4 8 3 2 6 4 7 8 0}, \\
1.53583516 & <C^{s}\left(S_{\frac{1}{4}}\right)<\mathbf{1 . 5 3 5 8 3 6 3 0} \\
1.5608170 & <C^{s}\left(S_{0.277}\right)<\mathbf{1 . 5 6 0 8 2 1 4} \\
1.56159516 & <C^{s}\left(S_{0.278}\right)<\mathbf{1 . 5 6 1 5 9 9 6 3} \\
1.543695 & <C^{1}\left(S_{\frac{1}{3}}\right)<\mathbf{1 . 5 4 3 7 1 1} \\
1.47119 & <C^{s}\left(S_{\frac{4}{10}}\right)<\mathbf{1 . 4 7 2 8 6 .}
\end{aligned}
$$

Observe that the above bounds suffice to prove the conjectural values proposed in [13]. In particular, the bounds obtained for the case $C^{1}\left(S_{1 / 3}\right)$ prove the conjecture $C^{1}\left(S_{1 / 3}\right) \simeq 1.543$. We would like to clarify that there was a minor error in [13] as, according to the algorithm's output in this case, the correct conjectured value is 1.543 and not 1.537 as was written in [13].

3. Planar Cantor type sets $K_{r}$.

Let $\left\{K_{r}\right\}_{r \in\left(0, \frac{1}{2}\right)}$ be the family of planar Cantor type sets defined by (30) and $s=-\frac{\log 4}{\log r}$. In [27] it is shown that

$$
C^{s}\left(K_{r}\right)=g_{5}(r) \text { where } g_{5}(r):=(2 \sqrt{2}(1-r))^{s}
$$

whenever $s \in(0,1),(1-r) r^{\frac{2 s-1}{1-s}} \geq 2$, and $\frac{3 r^{s}}{(1-r)^{s}} \leq 2^{-\frac{s}{2}}$.

These conditions hold for $r<r_{0}$ with $r_{0} \simeq 0.10832764$.

Table 8 shows the outcomes obtained applying Theorem 2 together with Algorithm 13 to the family $K_{r}$. Observe that the algorithm recovers the value given by (49) when $r<r_{0}$. In the examples in Table 8 where (49) cannot be rejected, $\tilde{\mathbf{m}}_{k}$ and $g_{5}(w)$ coincide to at least twelve decimal places. For $r>0.17$, (49) does not hold true and $g_{5}(r)$ is an upper bound for $C^{s}\left(K_{r}\right)$.

The bounds provided by Theorem 2 are:

$$
\begin{aligned}
1.36337287765281 & <C^{s}\left(K_{\frac{1}{100}}\right)<\mathbf{1 . 3 6 3 3 7 2 8 7 7 6 5 2 9 1} \\
1.57996258547383 & <C^{s}\left(K_{0.05}\right)<\mathbf{1 . 5 7 9 9 6 2 5 8 5 4 7 5 3 3}, \\
\mathbf{1 . 7 5 5 1 2 6 4 8 6 6 5} & <C^{s}\left(K_{\frac{1}{10}}\right)<\mathbf{1 . 7 5 5 1 2 6 4 8 8 9 2} \\
\mathbf{1 . 8 2 9 6 5 2 8 4 2 7} & <C^{s}\left(K_{\frac{1}{8}}\right)<\mathbf{1 . 8 2 9 6 5 2 8 6 7 3} \\
\mathbf{1 . 9 2 4 4 2 0 8 4} & <C^{s}\left(K_{0.16}\right)<\mathbf{1 . 9 2 4 4 2 1 2 0} \\
\mathbf{1 . 9 4 6 5 4 2 6} & <C^{s}\left(K_{0.17}\right)<\mathbf{1 . 9 4 6 5 4 3 4} \\
\mathbf{1 . 9 7 8 6 8 1 4 7} & <C^{s}\left(K_{0.2}\right)<\mathbf{1 . 9 7 8 6 8 5 3 8} \\
1.954256 & <C^{s}\left(K_{\frac{1}{4}}\right)<\mathbf{1 . 9 5 4 3 0 0} \\
\mathbf{1 . 6 1 1 1 8} & <C^{s}\left(K_{0.4}\right)<\mathbf{1 . 6 8 9 5 1}
\end{aligned}
$$

Finally, we remark that the rate of convergence given by Theorem 2 provides an estimate of $C^{s}\left(K_{r}\right)$ for the cases where we do not have a general formula with accuracy that varies from seven decimal places to one decimal places. 


\begin{tabular}{|c|c|c|c|c|c|c|c|c|}
\hline $\mathbf{r}<\mathbf{r}_{\mathbf{0}}$ & $\mathbf{s}$ & $\mathbf{q}$ & $\mathbf{k}_{\max }$ & $\mathcal{Q}$ & $\tilde{\mathbf{m}}_{k}=\mathbf{C}^{s}\left(\mathbf{K}_{\mathbf{r}}\right)=\mathbf{g}_{\mathbf{5}}(\mathbf{r})$ & $\epsilon_{\mathbf{k}}$ & $\mathbf{g}_{\mathbf{5}}(\mathbf{r})$ & $\mathbf{k}_{\mathbf{s t b}}$ \\
\hline 0.01 & $\frac{\log 4}{\log 100}$ & 1 & 7 & 1.01422 & 1.363372877653 & $4.25566 \times 10^{-14}$ & 1.363372877653 & 2 \\
\hline 0.05 & $-\frac{\log 4}{\log 0.05}$ & 1 & 10 & 1.05824 & 1.579962585475 & $7.45665 \times 10^{-13}$ & 1.579962585475 & 2 \\
\hline 0.1 & $\frac{\log 4}{\log 10}$ & 1 & 10 & 1.09286 & 1.755126487784 & $1.12992 \times 10^{-9}$ & 1.755126487784 & 2 \\
\hline $\mathbf{r} \geq \mathbf{r}_{0}$ & & & & & $\tilde{\mathbf{m}}_{k}, \mathbf{g}_{\mathbf{5}}(\mathbf{r}) \in \mathbf{I}_{\mathbf{k}_{\max }}$ & & & \\
\hline $1 / 8$ & $\frac{\log 4}{\log 8}$ & 1 & 10 & 1.10064 & 1.829652855011 & $1.22729 \times 10^{-8}$ & 1.829652855011 & 2 \\
\hline 0.16 & $-\frac{\log 4}{\log 0.16}$ & 1 & 10 & 1.09847 & 1.924421022097 & $1.74625 \times 10^{-7}$ & 1.924421022097 & 3 \\
\hline & & & & & $\tilde{\mathbf{m}}_{\mathbf{k}}, \mathbf{g}_{\mathbf{5}}(\mathbf{r}) \notin \mathbf{I}_{\mathbf{k}_{\max }}$ & & & \\
\hline 0.17 & $-\frac{\log 4}{\log 0.17}$ & 1 & 10 & 1.09465 & 1.946542971745 & $3.35957 \times 10^{-7}$ & 1.949655110042 & 3 \\
\hline 0.2 & $-\frac{\log 4}{\log 0.2}$ & 1 & 10 & 1.07339 & 1.978683424694 & $1.94598 \times 10^{-6}$ & 2.020532989127 & 3 \\
\hline $1 / 4$ & 1 & 1 & 10 & 1 & 1.954277821708 & $2.157919 \times 10^{-5}$ & 2.121320343560 & 9 \\
\hline 0.4 & $-\frac{\log 4}{\log 0.4}$ & 3 & 11 & 1.19455 & 1.650343901758 & $3.91609 \times 10^{-2}$ & 2.225958183662 & $>k_{\max }$ \\
\hline
\end{tabular}

Table 8: Planar Cantor type sets $\left\{K_{r}\right\}$

\begin{tabular}{|c|c|c|c|c|c|}
\hline $\mathbf{k}$ & $\mathbf{x}$ & $\mathbf{y}$ & $\mathbf{d}$ & $\tilde{\mathbf{m}}_{k}$ & $\mathbf{I}_{k}$ \\
\hline 5 & 0.55 & 0.091125 & 0.458875 & 1.02422358 & $(0.39037468,1.65807248)$ \\
\hline 6 & 0.55 & 0.111375 & 0.438625 & 1.03859290 & $(0.75336089,1.32382491)$ \\
\hline 7 & 0.56014905 & 0.11967877 & 0.44047028 & 1.03299380 & $(0.90463940,1.16134821)$ \\
\hline 8 & 0.44626331 & 0.00373669 & 0.44252661 & 1.03252769 & $(0.97476821,1.09028718)$ \\
\hline 9 & 0.55662225 & 0.11305651 & 0.44356574 & 1.03231740 & $(1.00632562,1.05830917)$ \\
\hline 10 & 0.55549190 & 1. & 0.44450810 & 1.03191238 & $(1.02021608,1.04360868)$ \\
\hline 11 & 0.55549190 & 0.99965949 & 0.44416759 & 1.03180195 & $(1.02653862,1.03706529)$ \\
\hline 12 & 0.55567918 & 1.00 & 0.44432082 & 1.03153497 & $(1.02916647,1.03390348)$ \\
\hline 13 & 0.55567918 & 1 & 0.44432082 & 1.03153497 & $(1.03046914,1.03260080)$ \\
\hline 14 & 0.55567918 & 1.0 & 0.44432082 & 1.03153497 & $(1.03105535,1.03201460)$ \\
\hline 15 & 0.44430686 & 0.888625 & 0.44431814 & 1.03152958 & $(1.03131375,1.03174542)$ \\
\hline 16 & 0.55568686 & 1. & 0.44431314 & 1.03151950 & $(1.03142237,1.03161663)$ \\
\hline 17 & 0.55568686 & 1 & 0.44431314 & 1.03151950 & $(1.03147579,1.03156321)$ \\
\hline 18 & 0.44431187 & 0.888625 & 0.44431313 & 1.03151949 & $(1.03149981,1.03153916)$ \\
\hline 19 & 0.44431244 & 0.888625 & 0.44431256 & 1.03151833 & $(1.031509482,1.03152719)$ \\
\hline 20 & 0.44431244 & 0.888625 & 0.44431256 & 1.03151833 & $(1.03151434,1.03152232)$ \\
\hline 21 & 0.44431244 & 0.888625 & 0.44431256 & 1.03151833 & $(1.03151654,1.03152013)$ \\
\hline
\end{tabular}

Table 9: Central Cantor set in the line $C_{0.45}$

Actually, in [13], 1.95 was proposed as a conjectural value for $C^{s}\left(K_{\frac{1}{4}}\right)$. Now, thanks to Theorem 2, we have proved this conjecture, for, by $(50)$ we have $C^{s}\left(K_{\frac{1}{4}}\right) \simeq 1.954$.

\subsection{The general case}

We present below Table 9 in order to illustrate the general case (see Section 2.4) for the centered Hausdorff measure. It gives the results for all iterations in the computation of $C^{s}\left(C_{0.45}\right)$ with $C_{0.45}$ being the central Cantor set in the line with contraction ratio 0.45 .

The columns in the table are: the iteration $k$, the center $x$ and the end point $y$ of the optimal ball, the radius $d=|x-y|$ of the optimal ball, the estimate $\tilde{m}_{k}$ of $C^{s}\left(C_{0.45}\right)$ at the $k$ th iteration, and the interval $I_{k}$ to which we can be sure that $C^{s}\left(C_{0.45}\right)$ belongs. Again, for simplicity all the values reported in the table are rounded to eight decimal places.

One can see that the values continue changing up to the limit of our computational power.

\section{Conclusions}

Research on the computability of metric measures on self-similar sets started in [17], ten years ago. The general method for the computation of metric measures was established in that paper, and a discussion on the computability of the existing metric measures was initiated. In the introduction of that paper one can read: 
"The exhaustive class of coverings used by this (the Hausdorff) measure gives it a special place as the smallest among all measures based on coverings. The price to be paid for such privilege is that the research on the exact Hausdorff measure of a self-similar set leads, with few exceptions, to the computation of bounds, but the exact Hausdorff measure of a self-similar set will remain unknown for some time."

In regard to the computation of the packing measure, there is written in [17]:

"Another consequence of these results is that the packing measure could be easier than the Hausdorff measure from a computational point of view . . . since the search for sets of optimal density is restricted to balls centered at E."

These predictions have been confirmed in the subsequent literature gathered in the bibliographical references below. Some results, already discussed earlier, on the exact packing and centered Hausdorff measures have been obtained using geometric methods, but the list of known results on Hausdorff measure has not grown significantly. The predictions in [17] have also been confirmed by the work on the computability of metric measures reported in [11], [12], [13], [14], and, in particular, by the results in the present paper, that permit a more detailed discussion of the frontiers of the computability of metric measures. Furthermore, something new can be added to the early expectations: by results in [12], [13], and the present paper, the centered Hausdorff measure and the packing measure can be added to the list of "computable" metric measures. This is fortunate, since a covering and a packing based measure are available for computation, at least for self-similar sets satisfying the SSC. As pointed out in Remark 4, this also gives additional valuable information on the spectrum of densities of $\mu$.

The above conclusions motivate a call to revisit well-established folklore on the topic of metric measures. Because of the double step definitions required for the packing and centered Hausdorff measures, it is a common opinion that these measures are too awkward to handle. However, in the setting of self-similar sets satisfying the OSC (and so also the $\mathrm{SSC}$ ), the second step in the definitions of $P^{s}$ and $C^{s}$ can be omitted (and, in the case of the packing measure, it can be omitted also in the more general setting of compact sets with finite packing measure). As shown in the present paper, one can take advantage of this fact for computational purposes, and it is now clear that the measures $P^{s}$ and $C^{s}$ will play a relevant role in the future, at least in computational issues.

We now discuss the frontiers of the computability of metric measures. At the present, two metric measures, $C^{s}$ and $P^{s}$, can be computed with the accuracy necessary for potential technical applications only in the case of self-similar sets satisfying the SSC and having small contraction ratios. In these cases, if the contraction ratios are small enough the results in this paper indicate that an optimal ball might exist, and a formula giving the exact value of the corresponding metric measure might be found by theoretical methods. Moreover, our algorithm is an efficient tool for identifying what might be the optimal ball, if the algorithm stabilizes at an early iteration. On the other hand, if the contraction ratios are large, then any attempted theoretical approach could be doomed to failure, since the problem of the calculation of $C^{s}$ and $P^{s}$ is essentially computational.

The bounds on the maximum error provided by Theorems 1 and 2 decrease exponentially with the number $k$ of iterations, but the number of calculations grows at a much faster rate when $k$ increases, since the number of feasible balls depends on the square of the number of points in $A_{k}$, and this in turn grows exponentially with $k$. In this regard, the reductions, obtained in [13] and [14], of the families of balls that need to be considered are crucial. However, if the contraction radii increase, two things occur that can render accurate computation of $P^{s}$ and $C^{s}$ impossible. First it is necessary to use smaller balls, since the minimum separation distance $c$ between the basic cylinders decreases, so the number of balls to be explored increases. Second and more important, in order to obtain good estimates of the $\mu$-measure of a ball, it is necessary to go to an iteration $k$ for which the size of the cylinders of the $k$ th generation is small relative to the size of the ball, and for this purpose we have to go to more advanced iterations for balls of small size. On the other hand, if the contraction ratios are large, $k$ must be taken to be still larger, so that the $k$-cylinders will be sufficiently small. In this case the computability of $P^{s}$ and $C^{s}$ encounters severe obstacles (see Remark 11).

Although the theoretical methods proposed in [17] can, in principle, be applied to self-similar sets satisfying the OSC, these cases are not yet amenable to computation by our method. This is because the OSC can be viewed as a limiting case, with the size of feasible balls going to zero (see [1] for an example in the line with the OSC, where the exploration of arbitrarily small balls must be undertaken in the search for a ball with maximal density) and the argument given above for cases satisfying the SSC and having large contraction ratios applies in an extreme form.

The computation of the spherical Hausdorff measure $H_{s p h}^{s}$ could still be feasible for the easier cases of small contraction ratios, but the results will be much poorer than those for $P^{s}$ or $C^{s}$ for increasing contraction ratios, because the class of balls centered at arbitrary points in the ambient space, which is the covering class used in the definition of $H_{s p h}^{s}$, is a much larger class than the covering classes used in the definitions of $P^{s}$ and $C^{s}$. Moreover, the computation of the Hausdorff measure is still unreachable by the argument given in [17].

Acknowledgement 17 The computational part of this work was performed in EOLO, the HPC of Climate Change of the International Campus of Excellence of Moncloa, funded by the MECD and MICINN. This is a contribution to the CEI Moncloa.

Part of the present work has been done while Marta Llorente was visiting Prof. Claude Tricot at the Mathematics department of the University Blaise Pascal. The author is grateful to Prof. Claude Tricot for valuable comments, fruitful discussions and hospitality. 


\section{References}

[1] Ayer, E. and Strichartz, R. (1999), Exact Hausdorff measure and intervals of maximal density for Cantor sets. Trans. Am. Math. Soc. 351, Num. 9, 3725-3741.

[2] Baek, H. K. (2006), Packing dimension and measure of homogeneous Cantor sets. Bull. Austral. Math. Soc. 74, no. $3,443-448$.

[3] Baoguo J. and Zhiwei Z. (2004), The packing measure of a class of generalized Sierpinski carpet. Analysis in Theory and Applications 20, 1, 69-76.

[4] Baoguo J., Zuoling Z., Zhiwei Z., and Jun L. (2003), The packing measure of the Cartesian product of the middle third Cantor set with itself. J. Math. Anal. Appl. 288, 424-441.

[5] Dai, M. and Tian, L. (2005), Exact Hausdorff centered measure of symmetry Cantor sets. Chaos Solitons Fractals 26 , no. $2,313-323$.

[6] Feng, D. (2003), Exact packing measure of linear Cantor sets. Math. Nachr. 248/249, 102-109.

[7] Feng D. and Hua J. G. (1998), Some relations between packing premeasure and packing measure. Bull. Lon. Math. Soc. 31, 665-70.

[8] Garcia, I. and Zuberman, L. (2012), Exact packing measure of central Cantor sets in the line. J. Math. Anal. Appl. 386, no. 2, 801-812.

[9] Hutchinson, J. E. (1981), Fractals and self-similarity. Ind. J. Math. 30, 713-747.

[10] Jia, B.; Zhou, Z. and Zhu, Z. (2002), A lower bound for the Hausdorff measure of the Sierpinski gasket. Nonlinearity 15, no. 2, 393-404.

[11] Llorente, M. and Morán, M. (2007), Self-similar sets with optimal coverings and packings. J. Math. Anal. Appl. 334, 1088-1095.

[12] Llorente, M. and Moran, M. (2010), Advantages of the centered Hausdorff measure from the computability point of view. Math. Scand. 107, no. 1, 103-122.

[13] Llorente, M. and Moran, M. (2012), An algorithm for computing the centered Hausdorff measures of self-similar sets. Chaos Solitons Fractals 45, no. 3, 246-255.

[14] Llorente, M. and Moran, M. (2014), Computability of the packing measure of totally disconnected self-similar sets. (To appear in ETDS).

[15] Mattila, P. (1982), On the structure of self-similar fractals. Ann. Acad. Sci. Fenn. Ser. A I, Math. 7, no. 2, $189-195$.

[16] Meinershagen, Sandra (2001/02), The Hausdorff measure and the packing measure on a perturbed Cantor set. Real Anal. Exchange 27 (2001/02), no. 1, 177-190.

[17] Morán M. (2005), Computability of the Hausdorff and packing measures on self-similar sets and the self-similar tiling principle. Nonlinearity 18, no. 2, 559-570.

[18] Saint Raymond, X. and Tricot, C. (1988), Packing regularity of sets in n-space. Math. Proc. Cambridge Philos. Soc. 103, no. 1, 133-145.

[19] Sullivan, D. (1984), Entropy, Hausdorff measures old and new, and limit sets of geometrically finite Kleinian groups. Acta Math. 153, no. 3-4, 259-277.

[20] Taylor, S. J. and C. Tricot (1985), Packing measure and its evaluation for a Brownian path. Trans. Amer. Math. Soc. 288, 679-699.

[21] Taylor, S. J. and C. Tricot (1986), Packing measure of rectifiable subsets of the plane. Math. Proc. Cambridge Philos. Soc. 99, 285-296.

[22] Tricot, C. (1979), Sur la classification des ensembles boréliens de mesure de Lebesgue nulle. These de doctorat, Geneve. 
[23] Tricot, C. (1982), Two definitions of fractional dimension. Math. Proc. Cambridge Philos. Soc. 91, 57-74.

[24] Tricot, C. (2008), Geometries et mesures fractales: Une introduction. France, Ellipses, 339 pp.

[25] Zhou Z. (2000), A new estimate of the Hausdorff measure of the Sierpinski gasket. Nonlinearity 13, 479-91.

[26] Zhu, Z. and Zhou, Z. (2002), The Hausdorff centred measure of the symmetry Cantor sets. Approx. Theory Appl. (N.S.) 18 , no. $2,49-57$.

[27] Zhu, Z. and Zhou, Z. (2008), The centered covering measures of a class of self-similar sets on the plane. Real Anal. Exchange 33, no. 1, 215-231. 